\title{
Mapping the subcortical connectivity of the human default mode network
}

\author{
Jian Li $\mathrm{i}^{\mathrm{a}, \mathrm{b}}$, William H. Curley ${ }^{\mathrm{a}, \mathrm{c}}$, Bastien Guerin ${ }^{\mathrm{b}, \mathrm{c}}$, Darin D. Dougherty ${ }^{\mathrm{b}, \mathrm{d}}$, Adrian V. Dalca ${ }^{\mathrm{b}, \mathrm{e}}$, Bruce Fischl ${ }^{\mathrm{b}, \mathrm{e}}$, Andreas \\ Horn $^{\mathrm{f}}$, Brian L. Edlow ${ }^{\mathrm{a}, \mathrm{b}, 1}$ \\ ${ }^{\text {a }}$ Center for Neurotechnology and Neurorecovery, Massachusetts General Hospital and Harvard Medical School, Boston, MA, USA \\ ${ }^{\mathrm{b}}$ Athinoula A. Martinos Center for Biomedical Imaging, Massachusetts General Hospital and Harvard Medical School, Charlestown, MA, USA \\ ${ }^{\mathrm{c}}$ Harvard Medical School, Boston, MA, USA \\ ${ }^{\mathrm{d}}$ Department of Psychiatry, Massachusetts General Hospital and Harvard Medical School, Boston, MA, USA \\ ${ }^{\mathrm{e}}$ Computer Science and Artificial Intelligence Laboratory, Massachusetts Institute of Technology, Cambridge, MA, USA \\ ${ }^{\mathrm{f}}$ Department of Neurology, Movement Disorders and Neuromodulation Sectio Charite, University Medicine Berlin, Berlin, Germany
}

\begin{abstract}
The default mode network (DMN) mediates self-awareness and introspection, core components of human consciousness. Therapies to restore consciousness in patients with severe brain injuries have historically targeted subcortical sites in the brainstem, thalamus, hypothalamus, basal forebrain, and basal ganglia, with the goal of reactivating cortical DMN nodes. However, the subcortical connectivity of the DMN has not been fully mapped and optimal subcortical targets for therapeutic neuromodulation of consciousness have not been identified. In this work, we created a comprehensive map of DMN subcortical connectivity by combining high-resolution functional and structural datasets with advanced signal processing methods. We analyzed 7 Tesla resting-state functional MRI (rs-fMRI) data from 168 healthy volunteers acquired in the Human Connectome Project. The rs-fMRI blood-oxygen-level-dependent (BOLD) data were temporally synchronized across subjects using the BrainSync algorithm. Cortical and subcortical DMN nodes were jointly analyzed and identified at the group level by applying a novel Nadam-Accelerated SCAlable and Robust (NASCAR) tensor decomposition method to the synchronized dataset. The subcortical connectivity map was then overlaid on a 7 Tesla 100 micron ex vivo MRI dataset for neuroanatomic analysis using automated segmentation of nuclei within the brainstem, thalamus, hypothalamus, basal forebrain, and basal ganglia. We further compared the NASCAR subcortical connectivity map with its counterpart generated from canonical seed-based correlation analyses. The NASCAR method revealed that BOLD signal in the central lateral nucleus of the thalamus and ventral tegmental area of the midbrain is strongly correlated with that of the DMN. In an exploratory analysis, additional subcortical sites in the median and dorsal raphe, lateral hypothalamus, and caudate nuclei were correlated with the cortical DMN. We also found that the putamen and globus pallidus are negatively correlated (i.e., anticorrelated) with the DMN, providing rs-fMRI evidence for the mesocircuit hypothesis of human consciousness, whereby a striatopallidal feedback system modulates anterior forebrain function via disinhibition of the central thalamus. Seed-based analyses yielded similar subcortical DMN connectivity, but the NASCAR result showed stronger contrast and better spatial alignment with dopamine immunostaining data. The DMN subcortical connectivity map identified here advances understanding of the subcortical regions that contribute to human consciousness and can be used to inform the selection of therapeutic targets in clinical trials for patients with disorders of consciousness.
\end{abstract}

\section{Introduction}

Recent advances in structural and functional connectivity mapping create opportunities for therapeutic neuromodulation of human brain networks (Horn and Fox, 2020). For patients with disorders of consciousness (DoC) caused by severe brain injuries, functional connectivity mapping can be used to identify widely connected network hubs that are therapeutic targets for stimulation (Edlow et al., 2020). The biological and mechanistic rationale for this targeted approach to neuromodulation has been demonstrated in rodent (Taylor et al., 2016) and non-human primate (Redinbaugh et al., 2020) models, which show that stimulation of subcortical network hubs promotes cortical reactivation and reemergence of consciousness from anesthetic coma. Emerging evidence from human electrical (Corazzol et al., 2017; Schiff et al., 2007; Thibaut et al., 2014), pharmacologic (Giacino et al., 2012; Whyte et al., 2014), and ultrasound-based (Cain et al., 2021a, 2021b; Monti et al., 2016) stimulation studies provide proof of principle that promoting recovery of consciousness in patients with DoC is possible (Edlow et al., 2021a). However, consensus on optimal subcortical therapeutic targets for neuromodulation of consciousness in humans has not been established (Edlow et al., 2021b).

A promising approach to identifying subcortical therapeutic targets is a "top-down" analysis of functional connectivity from canonical cortical networks that sustain consciousness. It is well established that the default mode network (DMN) contributes to self-awareness in the resting, conscious human brain (Buckner and DiNicola, 2019; Qin and Northoff, 2011; Raichle and Snyder, 2007). Although DMN functional connectivity alone is not sufficient for consciousness (Bodien et al., 2019; Demertzi et al., 2015; Norton et al., 2012), dynamic interactions between DMN nodes in the posterior cingulate, precuneus, medial prefrontal cortex, and inferior parietal lobules appear to be primary contributors to the neural correlates of consciousness (Bodien et al., 2017; Edlow et al., 2021a; Koch et al., 2016).

Given that the cortical nodes of the DMN are well-characterized and could be directly targeted with noninvasive methods such as transcranial direct current stimulation (tDCS) and transcranial magnetic stimulation (TMS), one could ask why the subcortical nodes of the DMN are clinically relevant. We could extend the question and ask why invasive stimulation methods such as deep brain stimulation (DBS) are not applicable at the cortical level (e.g., by placing a DBS electrode into the precuneus). Crucially, invasive methods have targeted subcortical regions for good reason, which has been referred to as the "funnel effect" of smaller brain nuclei (Parent and Hazrati, 1995). Projecting from cortical to subcortical structures (Swanson, 2000),

\footnotetext{
${ }^{1}$ Corresponding author. E-mail address: bedlow@mgh.harvard.edu (B. L. Edlow)
} 
information dimensionality (which is decompressed and openly available on cortical levels) is reduced (Bar-Gad et al., 2003). In ascending loops from subcortex to cortex, the reverse happens: information is expanded, decompressed, or de-referenced (Blouw et al., 2016). This architectural feature of the brain (Bota et al., 2015), which involves high-low-high dimensionality transforms of information, renders effects of neuromodulation on cortical versus subcortical levels strikingly different. Based on the large receptive and projective fields of subcortical brain structures, targeted neuromodulation of a small nucleus will affect a widely distributed and surprisingly large fraction of the entire cortex (Horn et al., 2019, 2017; Schiff et al., 2007). In contrast, diffuse neuromodulation techniques (e.g., TMS, tDCS), which modulate broad patches of cortical tissue, could have similar effects on networks (Fox et al., 2014). But doing the reverse (e.g., TMS to subcortical regions or DBS to cortical regions) would likely not produce the desired therapeutic effects. Hence, we believe it is crucial to precisely define subcortical nodes of the DMN to restore consciousness and cognitive function using targeted neuromodulation approaches such as DBS and low-intensity focused ultrasound pulsation (LIFUP).

Preliminary studies suggest that specific subcortical nuclei within the thalamus (Alves et al., 2019; Cunningham et al., 2017; Lee and Xue, 2018), basal forebrain (Alves et al., 2019), midbrain (Bär et al., 2016), pons (Fransson, 2005), and striatum (caudate and putamen) (Choi et al., 2012; Di Martino et al., 2008) are structurally and functionally connected to cortical DMN nodes. Testing for functional connectivity between subcortical regions and cortical DMN thus provides an opportunity to identify subcortical therapeutic targets in patients with DoC. Many such targets are amenable to therapeutic modulation by electromagnetic (Elias et al., 2020; Gratwicke et al., 2013; Kakusa et al., 2020; Schiff et al., 2007) and ultrasound-based therapies (Cain et al., 2021a, 2021b; Monti et al., 2016). However, DMN subcortical connectivity has not been fully mapped. In large part, this gap in knowledge is attributable to insufficient spatial resolution and low signal-to-noise ratio (SNR) of functional MRI, which poses a significant challenge to mapping functional connectivity for individual subcortical nuclei (Lee and Xue, 2018; Sclocco et al., 2018).

In this study, we aimed to create a comprehensive map of the subcortical connectivity of the DMN by combining high-resolution functional and structural datasets with advanced signal processing methods. Specifically, we used the resting-state functional MRI (rs-fMRI) dataset from 168 subjects acquired at 7 Tesla (7T) within the Human Connectome Project (HCP) (Smith et al., 2013). The rs-fMRI BOLD data were temporally synchronized across subjects using the BrainSync algorithm (Akrami et al., 2019; Joshi et al., 2018), which aligned all subjects' data into the same spatiotemporal space, making it possible to model brain networks as low-rank components. The cortical and subcortical data were jointly analyzed and a more complete DMN (which spans both cortex and subcortex) was identified at the group level by applying a novel Nadam-Accelerated SCAlable and Robust (NASCAR) tensor decomposition method (Li et al., 2019b, 2021). The subcortical functional connectivity map was then overlaid on the 7T 100 micron ex vivo MRI dataset (Edlow et al., 2019) for precise neuroanatomic analyses of the brainstem, thalamus, hypothalamus, and basal ganglia using the FreeSurfer segmentation atlas (Fischl, 2012), probabilistic thalamic segmentation atlas (Iglesias et al., 2018), the Harvard ascending arousal network atlas (Edlow et al., 2012), and the basal forebrain and hypothalamus atlas proposed in (Neudorfer et al., 2020).

We first tested the hypothesis that the central lateral nucleus (CL) of the thalamus and the ventral tegmental area (VTA) of the midbrain are strongly connected to cortical DMN. This hypothesis is based on evidence from anatomic connectivity studies (Alves et al., 2019; Morales and Margolis, 2017; Schiff, 2010, 2008; Yetnikoff et al., 2014), animal neuromodulation studies (Baker et al., 2016; Redinbaugh et al., 2020; Solt et al., 2014; Taylor et al., 2016), and limited human studies (Schiff et al., 2007), which collectively indicate that CL and VTA are widely connected subcortical network hubs whose stimulation may activate the cerebral cortex and promote reemergence of consciousness. Second, we performed exploratory analyses to identify additional subcortical regions whose BOLD signal shows strong positive or negative correlation (i.e., anti-correlation) with the cortical DMN, indicating that these regions could potentially be used as alternative targets of neuromodulation. Finally, we explored the functional connectivity differences between the NASCAR approach and the traditional seed-based method and compared the results to immunostain data from a human brainstem specimen. We release the subcortical DMN functional connectivity map via the Lead-DBS, FreeSurfer and OpenNEURO platforms for use in future neuromodulation studies.

\section{Materials and methods}

\subsection{In-vivo 7 T resting-state fMRI data}

We analyzed 7T resting-state fMRI (rs-fMRI) scans of healthy volunteers available from the Wash U/U Minn component of the Human Connectome Project (HCP) (Van Essen et al., 2012). We chose 7T, instead of 3T, dataset as it provides better SNR, particularly in subcortical regions. Eight subjects were excluded due to acquisition and/or preprocessing issues according to the HCP data release update (HCP Wiki, 2020), resulting in a total of 168 subjects used in this study. These 168 subjects were randomly split into two equally sized groups for reproducibility analysis. The following experiments were carried out on each group independently ( 84 subjects in each group). The rs-fMRI data were collected in four independent sessions with opposite phase encoding directions (PA, AP) using a gradient-echo EPI sequence ( $1.6 \mathrm{~mm}^{3}$ isotropic voxels, $\mathrm{TR}=1000 \mathrm{~ms}$, TE $\left.=22.2 \mathrm{~ms}\right)$, where each session was 15 mins long ( $T=900$ frames). Only the first session (PA) was used in this work to minimize the potential inter-subject misalignment due to the different EPI distortions in different phase encoding directions, although EPI distortion had been carefully corrected during the preprocessing (Smith et al., 2013). The analyses were performed on the HCP minimally preprocessed 7T rs-fMRI data (Glasser et al., 2013), which were resampled and coregistered onto a common atlas in MNI space. The data were then represented in a grayordinate system (Glasser et al., 2013), where there are approximately $32 \mathrm{~K}$ vertices on each hemisphere for cortical data and approximately $32 \mathrm{~K}$ voxels for subcortical data. No additional spatial smoothing beyond the standard minimal preprocessing pipeline ( $2 \mathrm{~mm}$ full width half maximum (FWHM) isotropic Gaussian smoothing) was applied, because linear smoothing often blurs boundaries between different functional regions (Bhushan et al., 2016; Li et al., 2018, 2020a; Li and Leahy, 2017), which is problematic in resolving the relationships between small subcortical sub-regions in the brainstem, thalamus, hypothalamus, and basal forebrain. 
bioRxiv preprint doi: https://doi.org/10.1101/2021.07.13.452265; this version posted July 14, 2021. The copyright holder for this preprint (which was not certified by peer review) is the author/funder, who has granted bioRxiv a license to display the preprint in perpetuity. It is made available under aCC-BY 4.0 International license.

\subsection{Inter-subject temporal synchronization}

Resting-state fMRI data are not directly comparable between subjects, as spontaneous BOLD activities in different subjects are not temporally synchronized. This is a critical issue even in stimulus-locked task fMRI data, where identical task design is used, because response latencies may differ between subjects (Friston et al., 1998). However, one of the assumptions in the low-rank tensor model we used in this work (described in the next section) is temporal synchrony across subjects, as the model does not work well on asynchronous fMRI data (Li et al., 2021). Therefore, we applied the BrainSync algorithm to achieve temporal alignment of the fMRI data (Joshi et al., 2018). BrainSync seeks an optimal temporal orthogonal transformation between two subjects, such that after synchronization the time series in homologous regions of the brain are highly correlated. In order to avoid the potential bias introduced by selecting any specific reference subject, we used the extended group BrainSync algorithm (Akrami et al., 2019) to build one virtual reference subject. This virtual reference subject is close, in the mean square sense, to all real subjects in the high dimensional
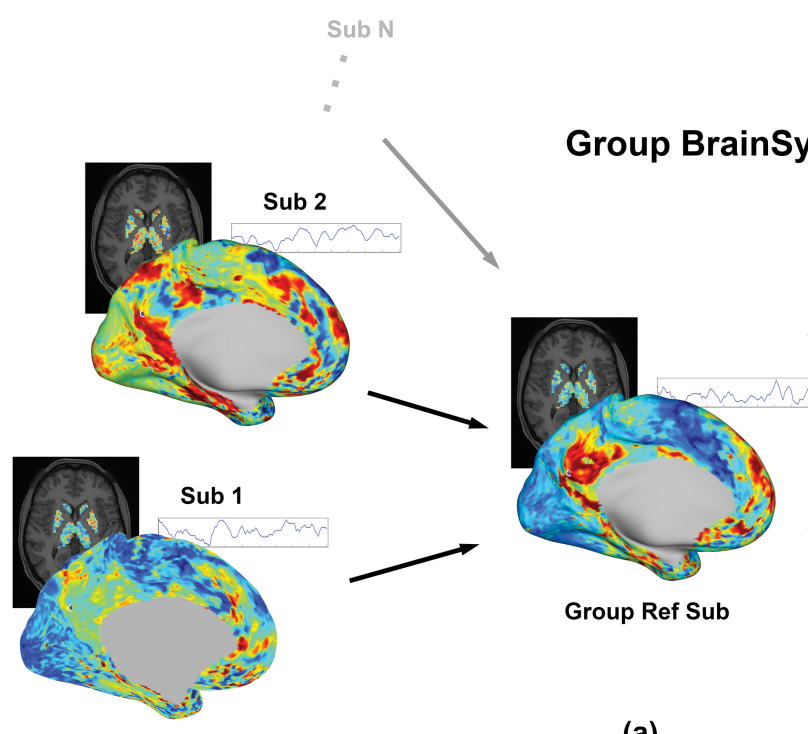

(a)

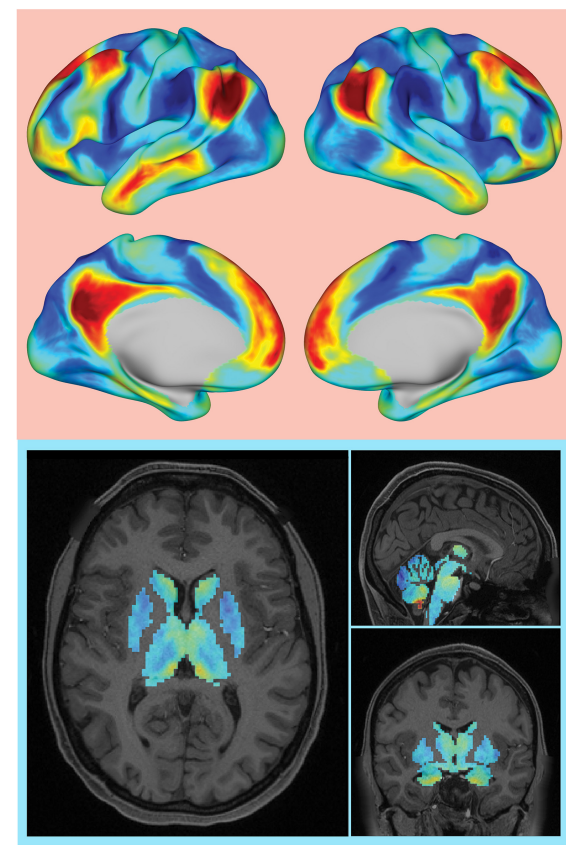

(e)

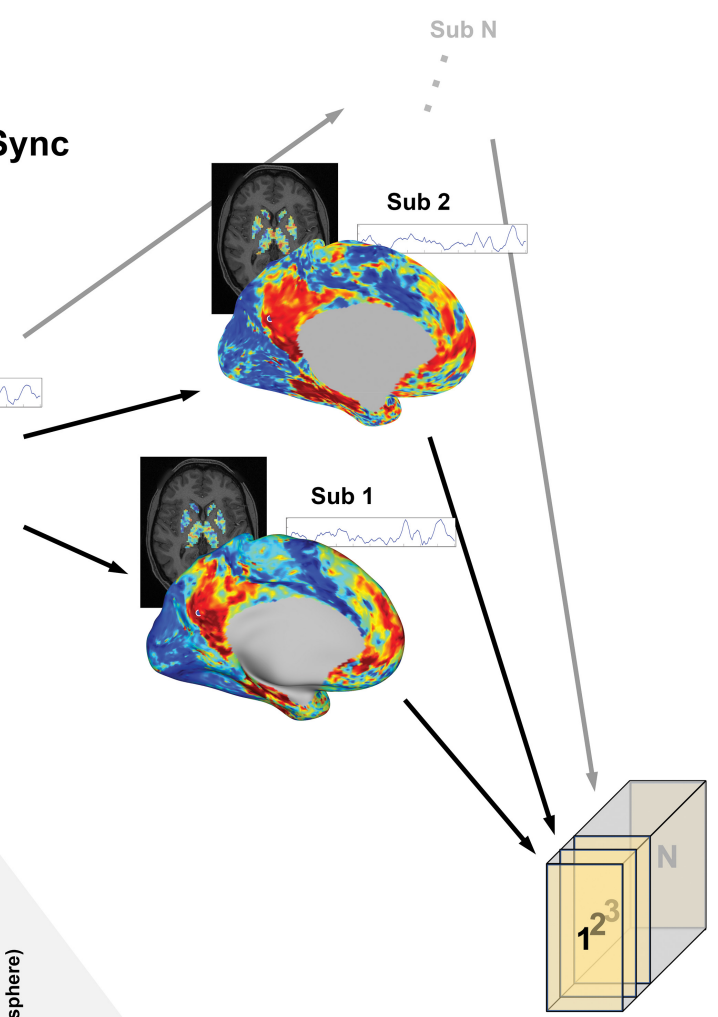

(b)

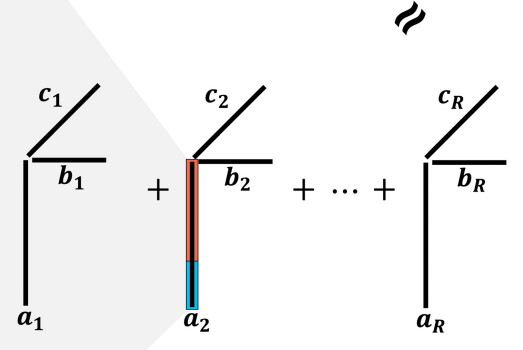

(c)

Fig. 1. Brain network identification pipeline. (a) Group BrainSync transform for temporal alignment; (b) 3D tensor formation (space x time $\mathrm{x}$ subject); (c) Tensor decomposition using the Nadam-Accelerated SCAlable and Robust (NASCAR) canonical polyadic decomposition. $\boldsymbol{a}_{i}, \boldsymbol{b}_{i}$, and $\boldsymbol{c}_{i}$ are the spatial map, the temporal dynamics, and the subject participation level for $i^{\text {th }}$ component, respectively; (d) Grayordinate representation of the spatial map of the default mode network (DMN); (e) The cortical map and the subcortical map of the DMN. 
space. Then we aligned all real subjects' data to that virtual reference to obtain a multi-subject synchronized dataset, Fig. 1 (a). Crucially, applying BrainSync will not alter functional connectivity metrics (as carried out by correlation coefficients across BOLD series) when calculated using the whole time period (Joshi et al., 2018).

\subsection{Tensor-based brain network identification}

Let $\boldsymbol{X} \in \mathbb{R}^{V \times T}$ be the synchronized rs-fMRI data of an individual subject, where $V$ is the number of vertices or voxels (space) and $T=900$ is the number of time points (time). All subjects were concatenated along the third dimension (subject), forming a data tensor $X \in \mathbb{R}^{V \times T \times S}$, where $S$ is the number of subjects, Fig. 1 (c). We model brain networks present in the group rs-fMRI data as a low-rank Canonical Polyadic (CP) model. Mathematically, the tensor $\boldsymbol{X}$ can be expressed as a sum of $R$ rank-1 components:

$$
\boldsymbol{x} \approx \sum_{r=1}^{R} \lambda_{r} \boldsymbol{a}_{r} \circ \boldsymbol{b}_{r} \circ \boldsymbol{c}_{r}
$$

where each rank-1 component $\lambda_{i} \boldsymbol{a}_{i} \circ \boldsymbol{b}_{i} \circ \boldsymbol{c}_{i}$ can be viewed as a brain network; $\boldsymbol{a}_{i} \in \mathbb{R}^{V}, \boldsymbol{b}_{i} \in \mathbb{R}^{T}$, and $\boldsymbol{c}_{i} \in \mathbb{R}^{S}$ are the spatial map, the temporal dynamics, and the subject participation level, respectively, in the $i^{\text {th }}$ network; $\lambda_{i}$ is the magnitude of that network, representing a relative strength of the activity in the $i^{\text {th }}$ network to other networks; "o" represents the outer product between vectors. $R$ is the desired total number of networks. We use $R=30$, as the rank of fMRI data has been shown to be limited (Calhoun et al., 2001; Li et al., 2021), and the network identification result is not sensitive to the choice of $R$ as we explain below (Li et al., 2019b, 2021).

We solved this network identification (tensor decomposition) problem (Eq. (1)) using the Nadam-Accelerated SCAlable and Robust (NASCAR) canonical polyadic decomposition algorithm (Li et al., 2019b, 2021). NASCAR employs an iterative method using low-rank solutions as part of the initializations when solving higher-rank problems. The robustness of the solutions to initializations and the choice of $R$ and the scalability to large dataset is substantially improved by using this warm start approach, and its superior performance over other traditional network identification methods has been demonstrated in applications to both electroencephalography (EEG) data (Li et al., 2019a, 2017) and fMRI data (Li et al., 2021, 2020b, 2019b).

\section{Table 1}

Subcortical regions of interest with respect to the FreeSurfer aseg altas (FreeSurfer), the probabilistic thalamic nuclei atlas (PTN), the Harvard ascending arousal network atlas (AAN), the basal forebrain / hypothalamus atlas $(\mathrm{BF} / \mathrm{HT})$, and their acronyms.

\begin{tabular}{|c|c|c|c|c|c|}
\hline FreeSurfer & PTN & Acronym & FreeSurfer & $\mathrm{BF} / \mathrm{HT}$ & Acronym \\
\hline \multirow[t]{25}{*}{ Thalamus } & Anteroventral & $\mathrm{AV}$ & \multirow[b]{16}{*}{ FreeSurfer } & Diagonal band of Broca & $\mathrm{dB}$ \\
\hline & Central Medial & $\mathrm{CeM}$ & & Nucleus Basalis of Meynert & NBM \\
\hline & Central Lateral & $\mathrm{CL}$ & & Medial Preoptic & MPO \\
\hline & Centromedian & $\mathrm{CM}$ & & Paraventricular & $\mathrm{Pa}$ \\
\hline & Lateral Dorsal & LD & & Dorsal Periventricular Hypothalamic & DPEH \\
\hline & Lateral Geniculate Nucleus & LGN & & Lateral Hypothalamus & $\mathrm{LH}$ \\
\hline & Lateral Posterior & LP & & Ventromedial Hypothalamic & VM \\
\hline & Limitans (Suprageniculate) & $\mathrm{LSg}$ & & Arcuate Hypothalamic & AN \\
\hline & Medial Dorsal lateral & MDl & & Periventricular Hypothalamic & $\mathrm{Pe}$ \\
\hline & Medial Dorsal medial & MDm & & Dorsomedial Hypothalamic & DM \\
\hline & Medial Geniculate Nucleus & MGN & & Supraoptic Hypothalamic & $\mathrm{SO}$ \\
\hline & Reuniens (Medial Ventral) & MVRe & & Suprachiasmatic Hypothalamic & $\mathrm{SC}$ \\
\hline & Paracentral & $\mathrm{Pc}$ & & Tuberomammillary Hypothalamic & $\mathrm{TM}$ \\
\hline & Parafascicular & $\mathrm{Pf}$ & & Posterior Hypotahalamic & $\mathrm{PH}$ \\
\hline & Pulvinar Anterior & $\mathrm{PuA}$ & & Anterior Hypothalamic Area & AHA \\
\hline & Pulvinar Inferior & $\mathrm{PuI}$ & & $\mathbf{A A N}$ & Acronym \\
\hline & Pulvinar Lateral & $\mathrm{PuL}$ & \multirow[t]{9}{*}{ Brainstem } & Dorsal Raphe & $\mathrm{DR}$ \\
\hline & Pulvinar Medial & $\mathrm{PuM}$ & & Median Raphe & MnR \\
\hline & Ventral Anterior & VA & & Periaqueductal Grey & PAG \\
\hline & Ventral Anterior Magnocellular & VAmc & & Ventral Tegmental Area & VTA \\
\hline & Ventral Lateral anterior & VLa & & Locus Coeruleus & $\mathrm{LC}$ \\
\hline & Ventral Lateral posterior & VLp & & Laterodorsal Tegmental & LDTg \\
\hline & Ventromedial & VM & & Midbrain Reticular Formation & $\mathrm{mRt}$ \\
\hline & \multirow[t]{2}{*}{ Ventral Posterolateral } & \multirow[t]{2}{*}{ VPL } & & Parabrachial Complex & PBC \\
\hline & & & & Pontis Oralis & $\mathrm{PnO}$ \\
\hline FreeSurfer & & Acronym & & Pedunculotegmental & PTg \\
\hline Caudate & & $\mathrm{Cd}$ & & & \\
\hline Putamen & & Put & & & \\
\hline Globus Pallidus & & GP & & & \\
\hline
\end{tabular}




\subsection{Visualization of subcortical DMN connectivity}

The DMN was identified as the second strongest network (second largest $\lambda$ value), with the "physiological" signal being the strongest network (see the Discussion section). This is expected, as the DMN has been shown to be the most prominently active (or stable) brain network at rest (Buckner and DiNicola, 2019; Raichle, 2015). In contrast to the traditional definition of the DMN where only cortical nodes are considered, we redefine the DMN as a functional brain network that spans both cortex and subcortex. To explore the DMN subcortical functional connectivity, the subcortical section of the spatial DMN map was separated from the cortical section using grayordinate indices, Fig. 1 (c) and (d). The cortical section of the DMN, which we refer to as the "cortical map", was plotted on the tessellated (inflated) surfaces for reference, as shown at the top of Fig. 1 (e). The subcortical counterpart was converted into a $3 \mathrm{D}$ volumetric representation in MNI space, referred to as the "subcortical map", and trilinearly interpolated into a 0.5 mm $^{3}$ isotropic resolution using the "mri_convert" tool in FreeSurfer (Fischl, 2012), as shown at the bottom of Fig. 1 (e). This interpolation procedure to higher resolution enabled both better visualization and the region-of-interest (ROI)-based analyses described below. Small ROIs in the atlases, such as $\mathrm{CL}$, could vanish if the atlases are downsampled to the rs-fMRI resolution.

For better visualization of subcortical functional connectivity, particularly in relation to subcortical anatomy, the subcortical map was overlaid on a 7T 100 micron resolution ex vivo MRI dataset (Edlow et al., 2019) for precise neuroanatomic analyses. This dataset was acquired using a customized 31-channel coil over 100 hours of scan time and was co-registered to the MNI space. A manual examination and minor registration adjustment were performed to account for the subtle difference in the MNI template used for registering functional data in the HCP pipeline and that used in registering the $100 \mu m$ structural dataset.

\subsection{Region-of-interest-based analysis}

To study the functional connectivity for each subcortical ROI, we performed segmentation of the subcortical structures on the minimally preprocessed (Glasser et al., 2013) T1-weighted image of a reference subject (HCP subject ID: 100610, the default 7T subject provided by the HCP) using the automated segmentation tool (aseg atlas) in FreeSurfer (Fischl, 2012). Further sub-division of the thalamus and segmentation of thalamic nuclei was performed using a probabilistic atlas (PTN atlas) (Iglesias et al., 2018). The default segmentation output was used for all thalamic nuclei except for CL. Considering the small size and irregular shape of the CL nucleus, the CL segmentation mask was obtained by thresholding its posterior probability map at $0.03\left(88^{\text {th }}\right.$ percentile). This threshold was estimated by visual inspection of the averaged CL map over $100 \mathrm{HCP}$ subjects so that the thresholded map accurately represents the shape and location of the CL nucleus. We used the Harvard ascending arousal network atlas (AAN atlas) (Edlow et al., 2012) for sub-division of brainstem nuclei, and an atlas proposed in (Neudorfer et al., 2020) for sub-division of basal forebrain and hypothalamus (BF/HT) nuclei. Labels of the AAN atlas were manually traced and provided in the MNI space (Edlow et al., 2012). Finally, all atlases were upsampled into a $0.5 \mathrm{~mm}^{3}$ isotropic resolution if the original atlases were in a lower resolution. Details about the ROIs with respect to the atlases are shown in Table 1.

\subsection{Quantitative analysis and hypothesis testing}

Because the rs-fMRI time series were normalized to have zero mean and unit norm during preprocessing to satisfy the requirement for inter-subject synchronization (Joshi et al., 2018), the absolute values in the identified DMN are less interpretable than the relative differences among ROIs. Therefore, to facilitate a meaningful quantitative interpretation, we performed a normalization at each voxel of in the subcortical map by the $95 \%$ quantile (a scalar) of the values in the cortical DMN map, top of Fig. 1 (e). Thus, the normalized subcortical map indicates how strong the subcortical DMN activity is relative to the cortical DMN activity. Here we use the word "activity" to indicate the signals/values in either the cortical DMN map or subcortical DMN map (Fig. 1 (e)) identified using the NASCAR method. For each ROI, we plotted the normalized values of the subcortical map within that ROI, using a violin plot.

To address whether CL is strongly connected to the cortical DMN, we statistically tested if the mean of the subcortical DMN map within CL is significantly higher than that within the entire thalamus (including CL), using a two-sample t-test. Considering the volumetric interpolation procedure used above and the spatial smoothness of the rs-fMRI data, correction of $p$-values for multiple comparison are necessary. Corrections were performed based on random field theory (Brett et al., 2003; Worsley et al., 1992), where the number of resolution element ("resel") was calculated based on the volume of each ROI and the FWHM of volumetric smoothing described in (Glasser et al., 2013). We similarly tested for VTA using this procedure, but with a comparison to the entire brainstem region (including VTA).

We then performed an exploratory study to test whether there are other candidate ROI(s) within the thalamus, hypothalamus, brainstem, and basal forebrain that have a strong connection to the cortical DMN, and thus could be used as subcortical targets for neuromodulation. We repeated the statistical testing above for all other ROIs defined in the PTN, AAN, and BF/HT atlas, with the proper correction for multiple comparisons as described above.

\subsection{Comparison to seed-based method}

We placed a single-vertex seed in the posterior cingulate cortex (PCC), which is one of the most commonly used seed locations in the DMN (Fox et al., 2005). For each voxel in the subcortical region, we then computed the Pearson correlation between that subcortical voxel and the seed, generating a seed-based subcortical functional connectivity map. Similar to the NASCAR analysis in Section 2.4, we visualized the result by overlaying it on the 100 micron structural dataset. We performed this seed-based analysis with and without global signal regression preprocessing. We repeated the above procedure using a second widely used single-vertex seed in the ventromedial prefrontal cortex (vmPFC). 
bioRxiv preprint doi: https://doi.org/10.1101/2021.07.13.452265; this version posted July 14, 2021. The copyright holder for this preprint (which was not certified by peer review) is the author/funder, who has granted bioRxiv a license to display the preprint in perpetuity. It is made available under aCC-BY 4.0 International license.

\subsection{Comparison of rs-fMRI results with brainstem immunostaining data}

To validate the results, we compared the subcortical maps from the NASCAR and seed-based correlation analyses with tyrosine hydroxylase immunostain data from a human brainstem specimen. The brainstem specimen was donated from a 53-year-old woman, with written informed consent from a surrogate decision-maker as part of an Institutional Review Board-approved protocol. Additional details regarding the patient's medical history, as well as the brainstem fixation and sectioning procedures, have been previously described, as this brainstem provided the basis for the Harvard AAN atlas used here (Edlow et al., 2012). For this analysis, we performed new tyrosine hydroxylase stains (rabbit polyclonal anti-tyrosine hydroxylase antibody; Pel-Freez Biologicals; Rogers AR) on tissue sections from the level of the caudal and rostral midbrain. Tyrosine hydroxylase stains dopamineproducing neurons, and thus was used as a reference standard for the accuracy of the VTA functional connectivity maps produced by the NASCAR and seed-based correlation analyses. The full tyrosine hydroxylase immunostaining protocol is available at https://github.com/ComaRecoveryLab/ Subcortical DMN Functional Connectivity and the stained tissue sections are available for interactive viewing at https://histopath.nmr.mgh. harvard.edu.

\subsection{Reproducibility Analysis}

We randomly split the 7T HCP rs-fMRI data into two halves and performed the same network identification procedure on these two independent datasets. The DMN was identified from each group and visually compared. Quantitatively, we also computed the Pearson correlation between the spatial map of the two identified DMNs.
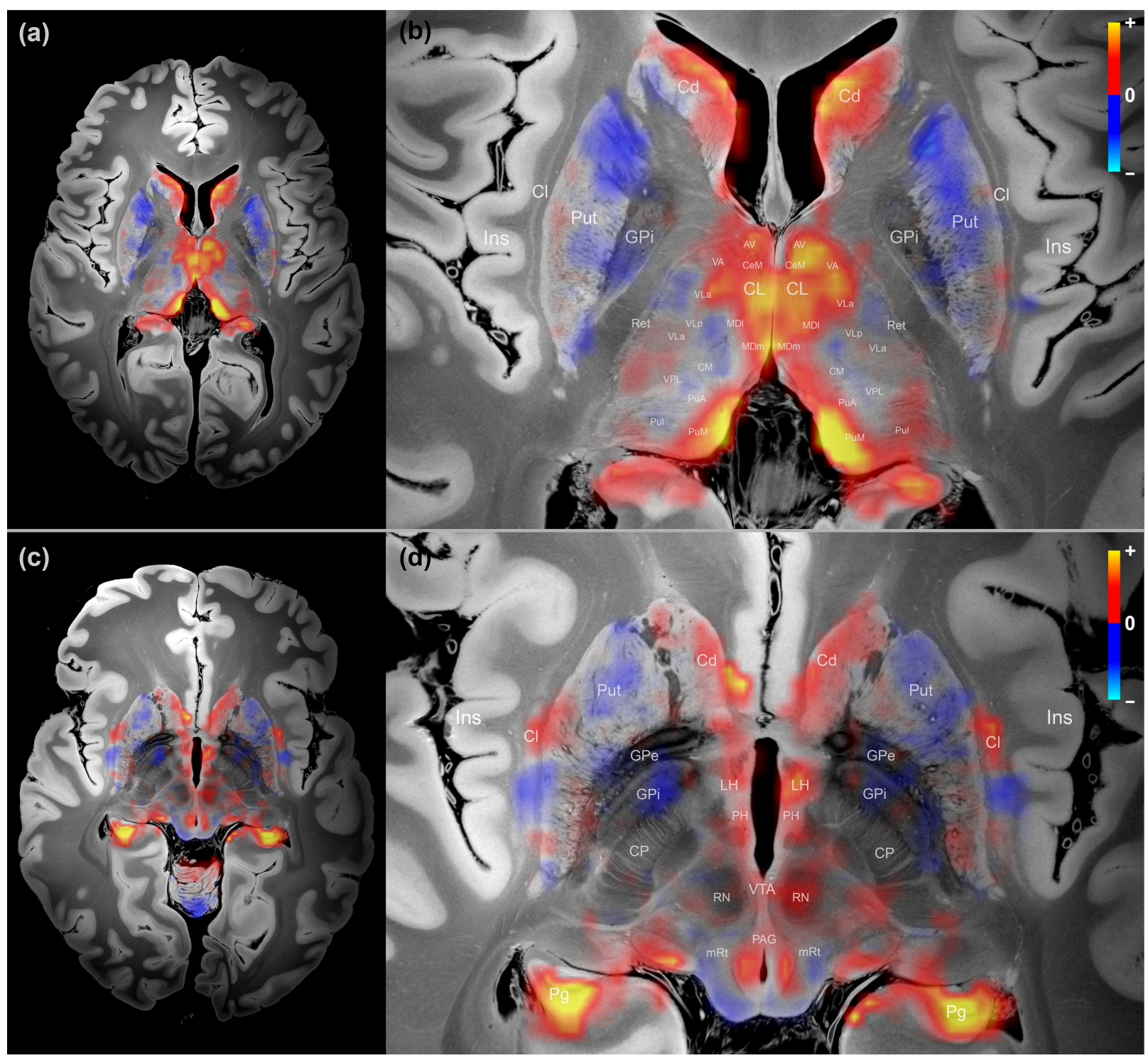

Fig. 2. Map of the subcortical DMN connectome. (a) Overview of thalamus and basal ganglia; (b) Zoom-in version of (a) with annotations; (c) Overview of rostral midbrain; (d) Zoom-in version of (c) with annotations. The warm color (yellow/orange) indicates positive association or correlation with the DMN, and the cold color (blue) indicates negative association or anti-correlation with the DMN. Cl - Claustrum; CP - cerebral peduncle; GPe - Globus Pallidus Externus; GPi - Globus Pallidus Internus; Ins - Insula; Pg - parahippocampal gyrus; Ret - Reticular nuclei; Refer to Table 1 for other acronyms. 
bioRxiv preprint doi: https://doi.org/10.1101/2021.07.13.452265; this version posted July 14, 2021. The copyright holder for this preprint (which was not certified by peer review) is the author/funder, who has granted bioRxiv a license to display the preprint in perpetuity. It is made available under aCC-BY 4.0 International license.

\section{Results}

\subsection{Qualitative visualization result}

Fig. 2 (a) and (b) show the DMN subcortical map identified by the NASCAR method in an axial slice through the thalamus and striatum (for visualization only, NASCAR was running on the entire grayordinate data). The DMN subcortical map at the level of the basal forebrain, hypothalamus, and rostral midbrain is shown in Fig. 2 (c) and (d). Fig. 3 shows the DMN subcortical map at the level of the caudal midbrain in (a), (b), and the rostral pons in (c), (d). We use "correlation" hereafter to indicate the functional connectivity relationship of the subcortical regions to the cortical DMN. However, this "correlation" is not the Pearson correlation coefficient (see Section 3.3). Rather, it represents the strength of the DMN activity at each subcortical region. The higher the magnitude of the value in a subcortical region (the actual value could be either positive or negative), the stronger "resonance" of this region to the cortical DMN. The entire 3D volumetric results are shown as a video in the supplementary material and available at https://github.com/ComaRecoveryLab/Subcortical_DMN_Functional_Connectivity. The visualizat-ion results are not sufficient for making inferences at individual voxels due to the low spatial resolution of the fMRI data, as well as imperfect inter-subject coregistration (more detail in the Discussion section). Supplementary Fig. S1 includes the DMN functional connectivity map in its native resolution overlaid on the same 100 micron structural MRI for reference.

Overall, we observed that the DMN subcortical components were largely symmetric about the midline of the brain, and the patterns appeared as spatially contiguous blobs. Importantly, there was no spatial constraint in the low-rank model itself, as shown in Fig. 1 (d), where each voxel/vertex was treated independently as part of the grayordinate representation during the decomposition. In other words, a random shuffle of the vertices/voxels
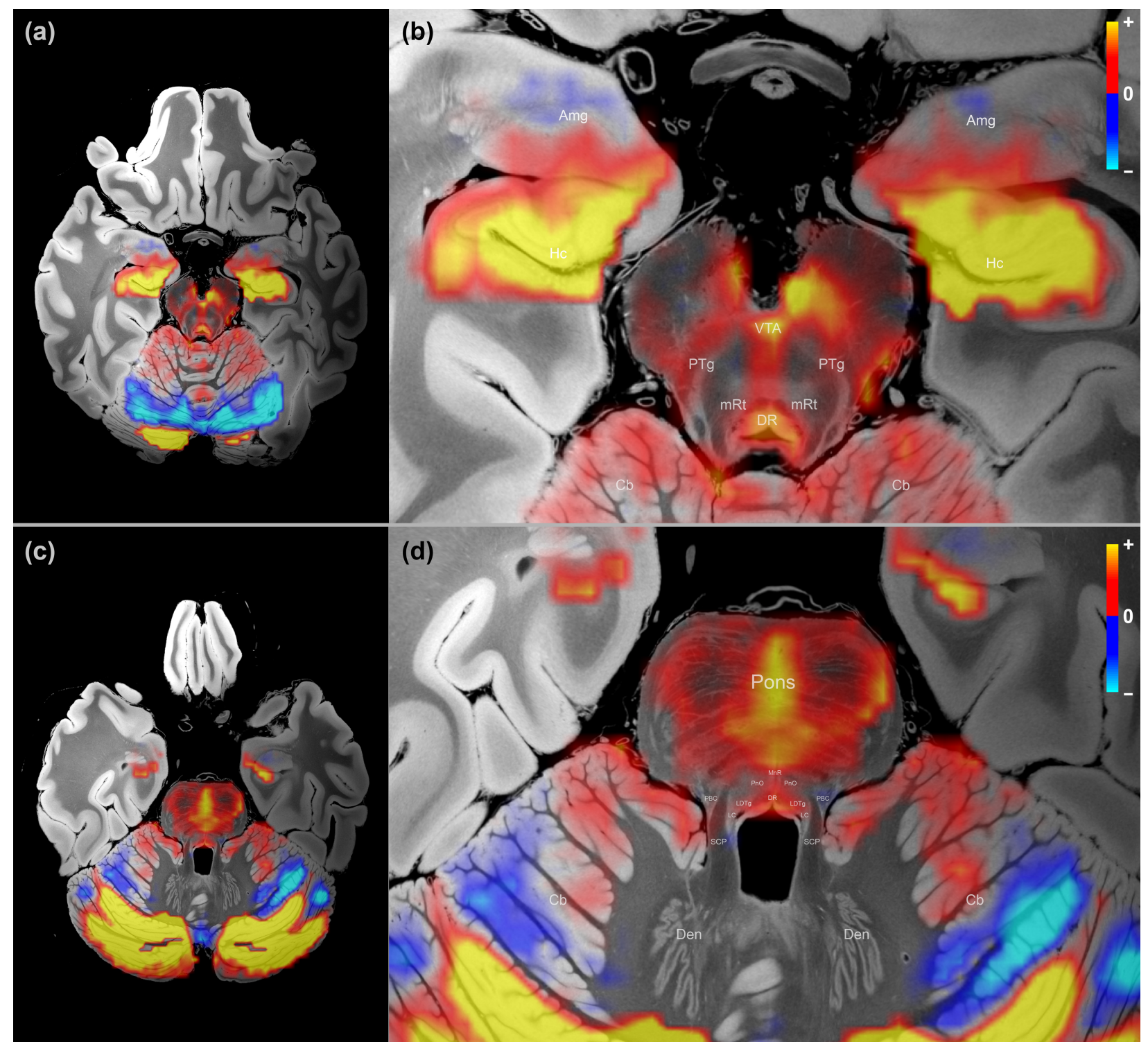

Fig. 3. Map of the subcortical DMN connectome. (a) Overview of caudal midbrain; (b) Zoom-in version of (a) with annotations; (c) Overview of rostral pons; (d) Zoomed version of (c) with annotations. See Fig. 2 for color scheme. $\mathrm{Cb}$ - cerebellum; Den - dentate nucleus of the cerebellum; SCP - superior cerebellar peduncle. Refer to Table 1 for other acronyms. 


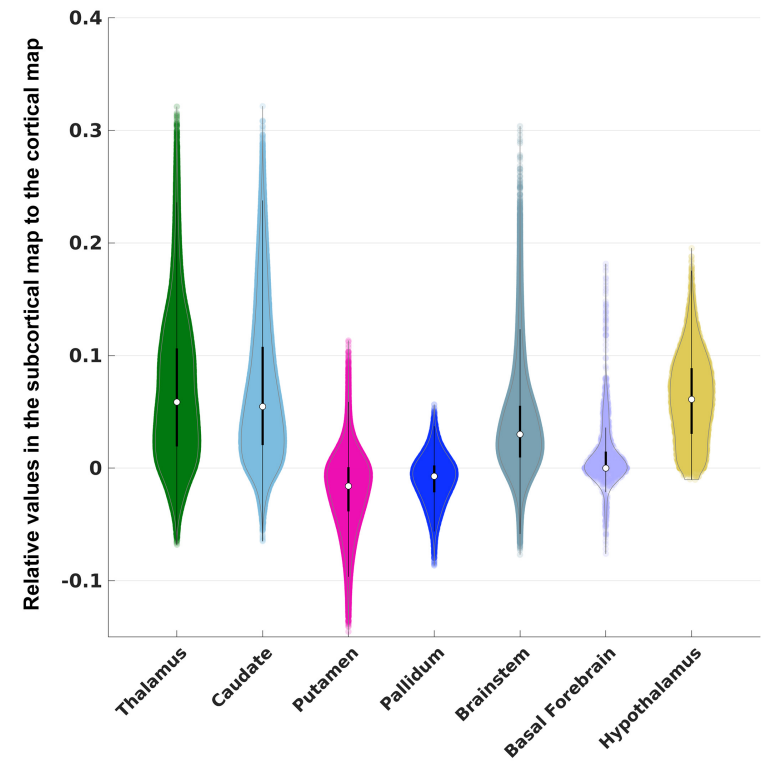

Fig. 4. Violin plots for large-scale ROIs defined in FreeSurfer aseg atlas. Each violin plot shows the distribution of the DMN signals overlaid with dots for each individual voxel. The white dot indicates the median statistics and the black bar through the white dot is the traditional boxplot, where the thicker bar represents the $25 \%$ to $75 \%$ quantile and the thinner bar represents the whisker length that is 1.5 times of the interquartile, covering approximately $99.3 \%$ of the data range. The color scheme of the violin plots follows that in the atlas. before decomposition and a corresponding shuffle in the reverse order after the decomposition would not change the results, indicating that these resulting patterns likely reflect a physiological property of the data, not an artifact of the processing pipeline.

With respect to the neuroanatomic localization, the strongest regions of subcortical DMN functional connectivity were observed within the central thalamus, lateral hypothalamus, caudate nucleus, ventral tegmentum of the midbrain, periaqueductal grey area of the midbrain, and midline raphe of the midbrain and pons. All of these regions have animal or human evidence supporting their roles in the modulation of arousal, and hence consciousness (Alam et al., 2002; Eban-Rothschild et al., 2016; Lu et al., 2006; Parvizi, 2001; Van der Werf et al., 2002; Villablanca et al., 1976). The subcortical regions that showed the strongest anticorrelations with the DMN were the putamen and globus pallidus interna, regions that constitute the inhibitory component of a mesocircuit that was postulated to modulate the cerebral cortex via GABAerigc innervation of the central thalamus (Schiff, 2010). The basal forebrain did not contain large clusters of correlated or anti-correlated voxels.

\subsection{Quantitative analysis result}

Fig. 4 displays results of the analysis of subcortical functional connectivity with the DMN, using subcortical structures defined in the FreeSurfer aseg atlas. Subcortical regions are displayed along the $\mathrm{x}$-axis, and the $y$-axis represents the normalized values with respect to the cortical DMN. We found that all subcortical structures exhibited substantially lower DMN activity compared to the cortex, with an averaged absolute percentage of $3.9 \%$. We observed that the highest subcortical DMN signal is in the thalamus, caudate, and brainstem, reaching approximately $30 \%$ of the cortical DMN signal strength. The thalamus, caudate, brainstem, and hypothalamus showed strong positive correlations with the DMN, and the basal forebrain showed moderate positive correlations with the DMN, although negative correlations were also observed in these regions. Interestingly, the majority of voxels within the globus pallidus and putamen exhibited negative correlations with the DMN.

Fig. 5 shows the violin plots of the DMN signals for the thalamic nuclei. CL showed significantly higher functional connectivity with the cortical $\mathrm{DMN}$ than the average of thalamic signals. CL had the $4^{\text {th }}$ highest median value (slightly higher median values were observed in AV, MDm, and PuM),

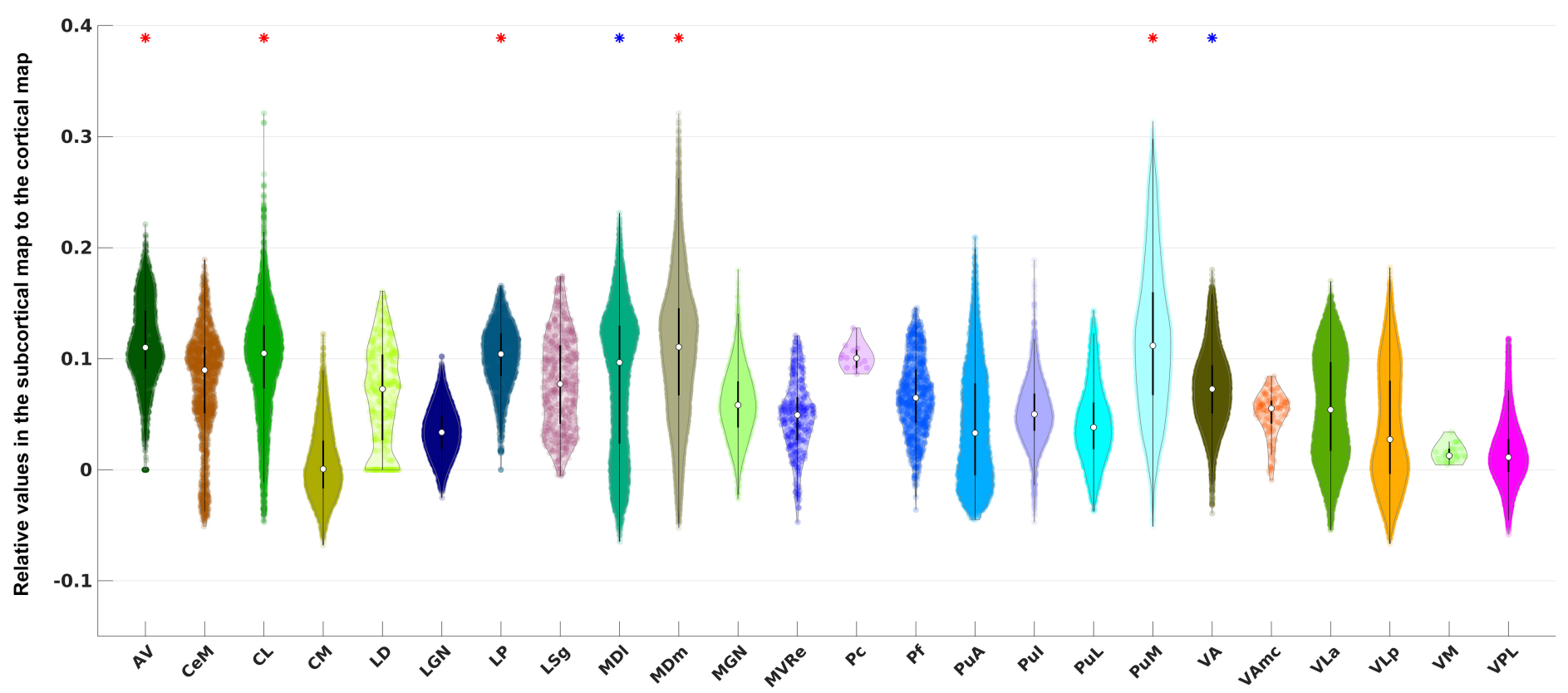

Fig. 5. Violin plots for thalamic ROIs defined in the PTN atlas. The acronyms of the nuclei are labeled along the $\mathrm{x}$-axis (see Table 1 for details). A star is placed above the violin plot if the average DMN signal within that nucleus is significantly higher than the mean signal of the entire thalamic region according to two sample Students' t-test. The star is colored in blue if the $p$-value is below the standard $\alpha$ cutoff value of 0.05 after the correction and colored in red if the $p$-value is below an $\alpha$ cutoff value of 0.001 (this cutoff was chosen heuristically for contrasting and highlighting a higher significance). 


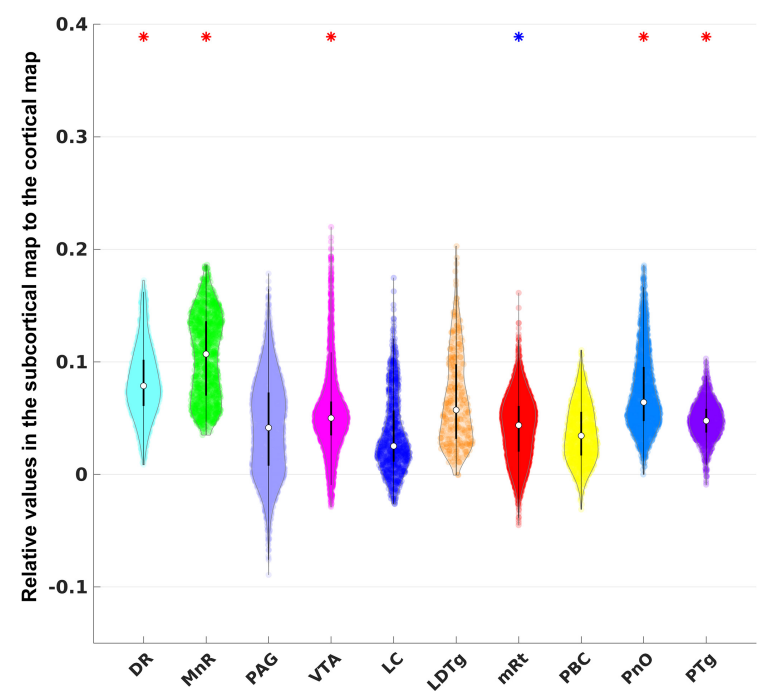

Fig. 6. Violin plots for brainstem ROIs defined in the AAN atlas. See Fig. 5 for interpretation of the statistical significance.

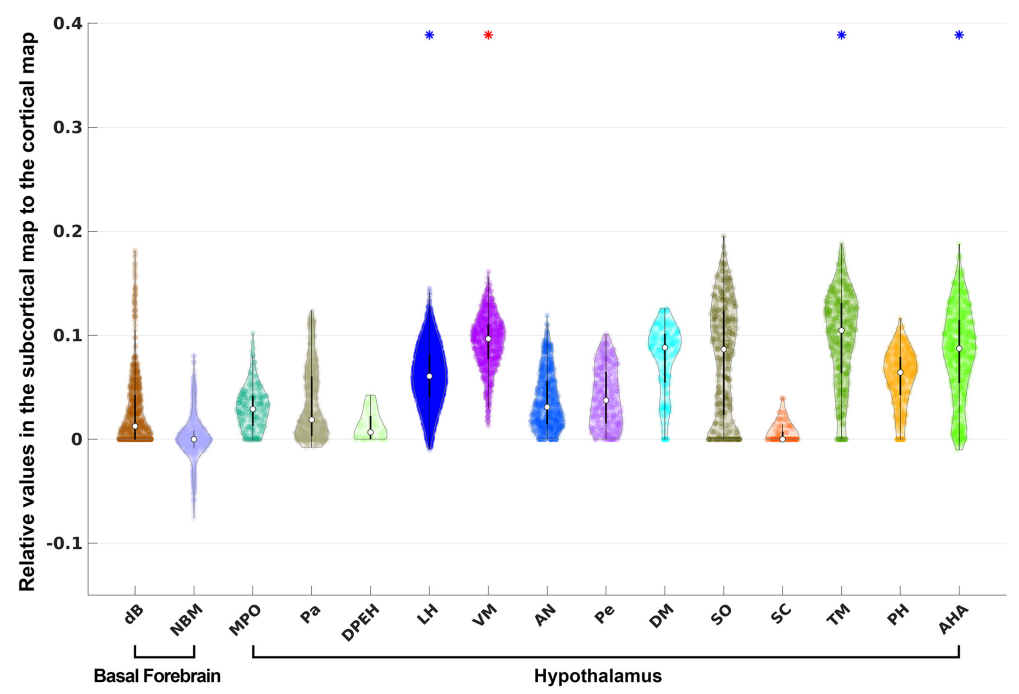

Fig. 7. Violin plots for hypothalamus and basal forebrain ROIs defined in the atlas of Neudorfer et al., 2020. See Fig. 5 for interpretation of the statistical significance.

and the highest maximum value among all thalamic nuclei. CL contained voxels with the strongest correlation to the DMN, reflected by the heavy tail on top of its distribution.

Fig. 6 shows similar violin plots for the brainstem region. We found that the VTA had significantly higher DMN functional connectivity than the average of the brainstem, consistent with previous studies (Bär et al., 2016). Although VTA did not show the highest median value, it did contain the highest maximum value among all brainstem nuclei.

In the exploratory analysis, we identified multiple additional nuclei that showed strong connection to the cortical DMN: AV, LP, MDl, MDm, PuM, and VA in the thalamus (Fig. 5), DR, MnR, mRt, PnO, and PTg in the brainstem (Fig. 6), and LH, VM, TM, and AHA in the hypothalamus (Fig. 7). Interestingly, distinct connectivity patterns were observed in $\mathrm{dB}$ and NBM, the two basal forebrain ROIs. Whereas dB showed exclusively positive DMN correlations, NBM showed a distribution of positive and negative correlations, yielding a median DMN connecting value close to zero. NBM was the only basal forebrain or hypothalamic nucleus with a substantial proportion of voxels showing negative correlations (i.e., anti-correlations) with the DMN.

\subsection{Seed-based method and immunostaining images}

Fig. 8 shows the seed-based correlation result for PCC in (a), (c) and for vmPFC in (b), (d) without global signal regression. The corresponding counterparts with global signal regression are shown in (e) - (h). Overall, these seed-based correlation results exhibit similar subcortical connectivity patterns (relative contrast between regions) to the patterns identified by NASCAR shown in Fig. 2 and Fig. 3. Specifically, in both the seed-based and NASCAR analyses, CL and VTA visually had the strongest correlation to the cortical DMN in comparison with other nuclei. However, the absolute correlation profile, especially the sign of the correlation (positively correlated vs anti-correlated), substantially differed between the results with global signal regression and in the ones without global signal regression. In the top row of Fig. 8, where no global signal regression had been applied, positive correlations were observed for most regions. This inflation of the correlation may be due to the global "physiological" or "vascular" component present in the fMRI data (Murphy and Fox, 2017; Zhu et al., 2015). In contrast, this "physiological" component was captured by NASCAR as a component separate from the DMN; thus, negative connections are clearly visible in the NASCAR result shown in Fig. 2 and Fig. 3 . Although global signal regression can be used in seed-based analyses to reduce contamination from the "physiological" component, as shown in the bottom row of Fig. 8, it can be difficult to interpret the meaning of these negative correlations, as it has been shown mathematically that global signal regression introduces negative correlation into the seed-based correlation results (Murphy et al., 2009; Murphy and Fox, 2017). Indeed, the co-existence of both positive and negative correlation after the global signal regression in the dopaminergic VTA region, as shown in Fig. 8 (g) and (h), does not appear to be anatomically consistent with prior anatomic atlases (Edlow et al., 2012; Trutti et al., 2021), with our immunostain data (Fig. 9), or with prior neuronal labeling studies in rodents, non-human primates, and humans (Breton et al., 2019; Root et al., 2016; Taylor et al., 2014), as discussed below. Finally, there were substantial variations in the seed-based correlation result depending on the choice of the seed point, which is consistent with findings in the literature (Uddin et al., 2009). For example, negative correlations were observed in the putamen and globus pallidus when PCC was selected as the seed shown in Fig. 8 (a), whereas they were barely visible when vmPFC was used as the seed shown in Fig. 8 (b).

\subsection{Reproducibility Analysis}

The spatial maps of the identified DMN from the two independent groups were visually indistinguishable, and the correlation coefficient between the two spatial maps (grayordinate representation) was 0.987 , demonstrating the high reproducibility of the results using our tensor-based analysis pipeline. 


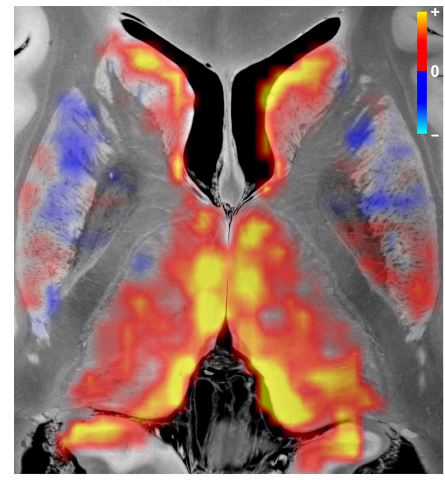

(a)

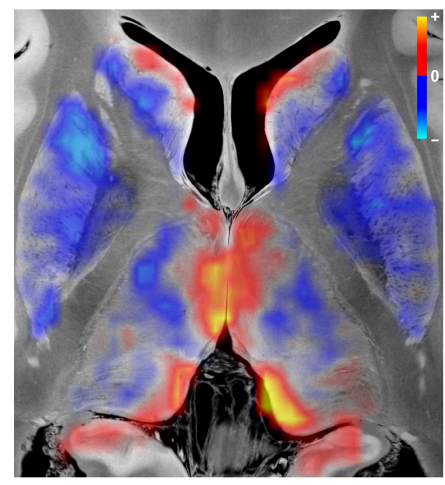

(e)

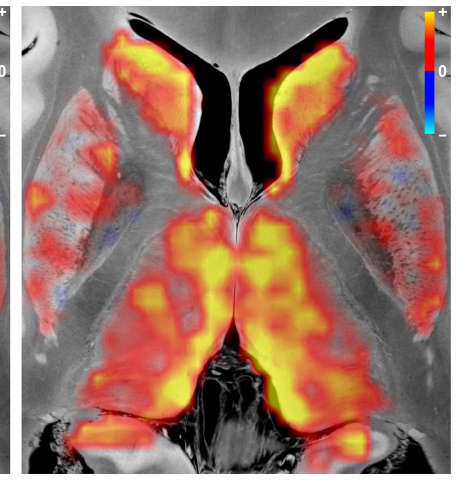

(b)

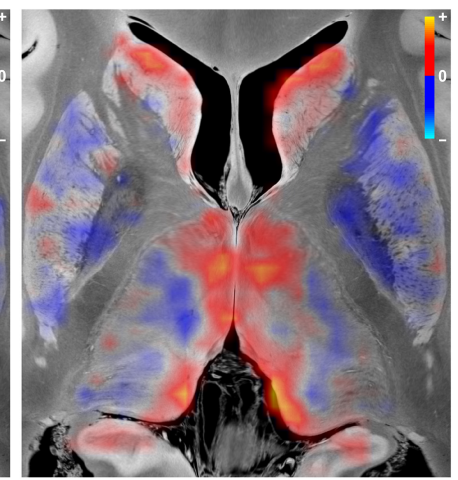

(f)

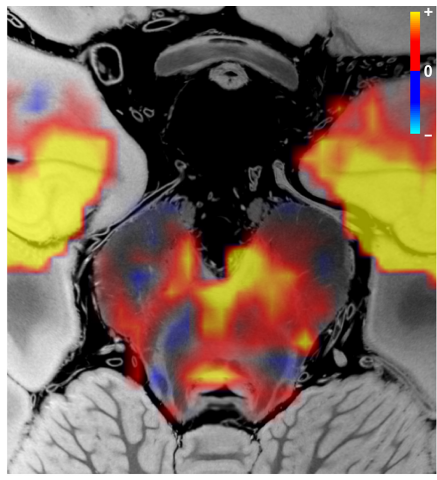

(c)

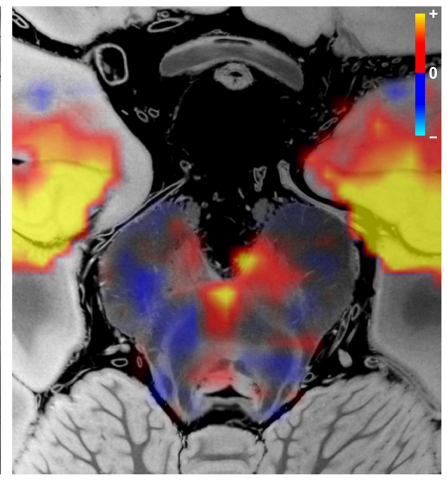

(g)

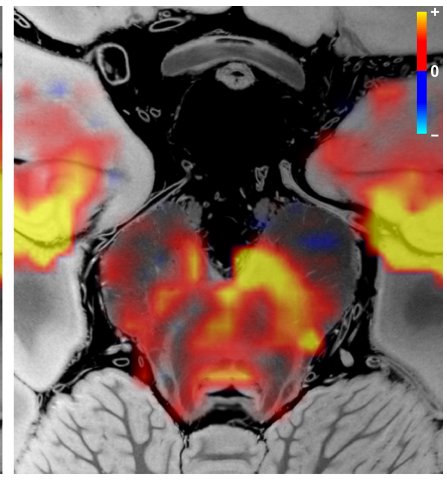

(d)

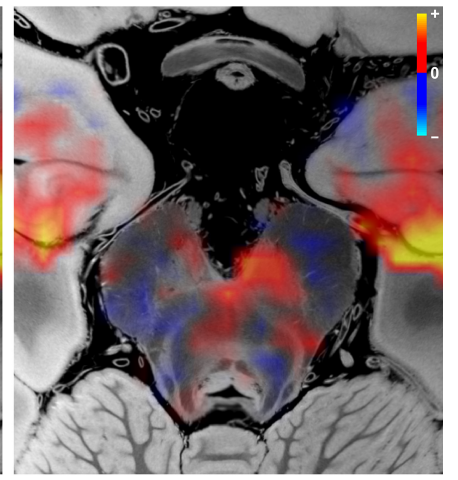

(h)

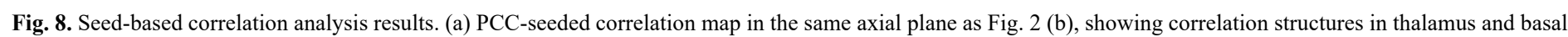

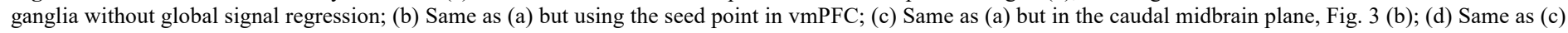
but using the seed point in vmPFC; (e) - (h) Same as (a) - (d) but with global signal regression.

\section{Discussion}

In this brain mapping study using 7T resting-state fMRI data from the HCP, we characterized the subcortical connectivity of the human DMN and openly release the results in standardized form (via Lead-DBS, OpenNEURO, and FreeSurfer). We expanded the DMN to include subcortical nodes by a joint analysis on the grayordinate system, providing a more complete map of the human DMN. The map was generated by a tensor-based NASCAR decomposition method that revealed extensive interconnections between the canonical cortical DMN and subcortical regions within the brainstem, thalamus, hypothalamus, basal forebrain, and basal ganglia. Further, the NASCAR and seed-based correlation results supported our hypothesis that CL and VTA are subcortical nodes strongly connected to the cortical DMN. The spatial, temporal, and physiological properties (e.g., correlations versus anti-correlations) of the subcortical DMN connectivity map create new opportunities to elucidate subcortical contributions to human consciousness and provide therapeutic targets for interventions that aim to promote recovery of consciousness in patients with severe brain injuries.

Our functional connectivity results are consistent with, and build upon, decades of electrophysiological and neuroimaging investigations of the subcortical networks that modulate consciousness. For example, the CL nucleus of the thalamus is a well-established hub node of reciprocal thalamocortical networks, as CL is richly innervated by arousal neurons of the brainstem and basal forebrain (Steriade and Glenn, 1982) and provides diffuse innervation of the neocortex (Dempsey and Morison, 1941). More recently, deep brain stimulation studies targeting CL in non-human primates (Baker et al., 2016; Redinbaugh et al., 2020) and humans with severe brain injuries (Schiff et al., 2007) have confirmed the role of CL in modulating consciousness. However, a non-invasive, fMRI-based biomarker of CL functional connectivity has been elusive, and the mechanisms and pathways by which the central thalamus modulates the DMN remain an area of active study (Cunningham et al., 2017; Guldenmund et al., 2013; Wang et al., 2014). Here, we provide robust evidence, with out-of-sample testing, for strong positive correlations between the CL nucleus and the human DMN. Indeed, individual voxels within CL showed the strongest correlation with the DMN of any thalamic voxels, and the median correlation strength of CL voxels with the DMN was the $4^{\text {th }}$ highest of all thalamic nuclei. These observations suggest that high spatial and temporal resolution rs-fMRI with advanced signal processing and modeling methods, as performed here, provide a potential biomarker of CL-DMN functional connectivity and an opportunity to test the hypothesis that CL stimulation induces reemergence of consciousness via CL-DMN functional connectivity in patients with severe brain injuries.

The potential translational impact of the CL-DMN functional connectivity findings is particularly noteworthy when considered in the context of the mesocircuit hypothesis of consciousness, for which we provide new rs-fMRI functional connectivity evidence in the human brain. Specifically, we observed anti-correlations between the putamen, globus pallidus, and the DMN, indicating that putaminal and pallidal activity toggles inversely with 


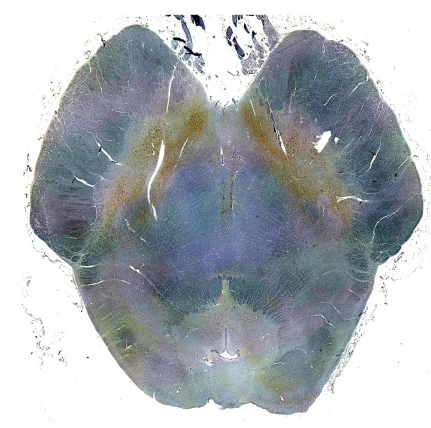

(a)

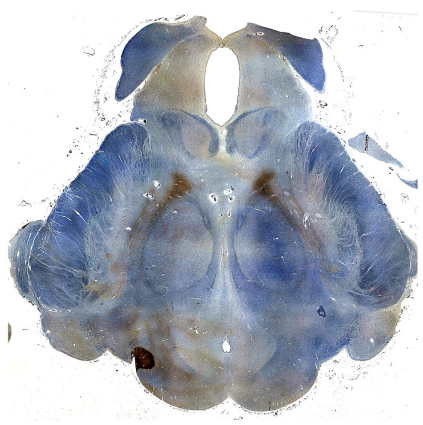

(b)
Fig. 9. Immunostain images of dopaminergic ventral tegmental area (VTA) neurons in (a) the caudal midbrain and (b) the rostral midbrain. Dopaminergic neurons (brown) were immunostained with tyrosine hydroxylase, and each axial section was counterstained with hematoxylin (blue). In both the caudal and rostral midbrain sections, the VTA neurons extend laterally and posteriorly along the lateral border of the decussation of the superior cerebellar peduncles (a) and the red nuclei (b). The human brainstem specimen used for these immunostains was donated by a 53-year-old woman who died of non-neurological causes. A surrogate-decision maker provided written informed consent for brain donation and postmortem research. Additional details about the specimen have been previously published (Edlow et al. 2012). central thalamus (Cain et al., 2021a; Monti et al., 2016) in patients with disorders of consciousness.

Additional neuroanatomic insights provided by the subcortical DMN connectivity map include new evidence for brainstem nodes that are strongly connected to the DMN. The VTA functional connectivity findings confirmed our hypothesis that the VTA is strongly connected to the DMN, consistent with recent rs-fMRI evidence for VTA functional connectivity with the posterior cingulate/precuneus, a central hub node of the DMN (Buckner and DiNicola, 2019; Thomas Yeo et al., 2011). VTA modulation of consciousness via dopaminergic signaling has been suggested by preclinical studies using pharmacologic (Kenny et al., 2015; Solt et al., 2011), electrical (Solt et al., 2014), optogenetic (Eban-Rothschild et al., 2016; Taylor et al., 2016), and chemogenetic (Oishi et al., 2017) stimulation, as well as a mouse dopamine knock-out model (Palmiter, 2011). However, until recently, there has only been indirect evidence for dopaminergic VTA modulation of human consciousness from pharmacological studies using dopaminergic drugs (Fridman et al., 2019, 2010; Giacino et al., 2012), as well as positron emission tomography studies of dopamine receptor dynamics (Fridman et al., 2019). Now, with emerging evidence for dopaminergic VTA modulation of human consciousness via DMN functional connectivity, there is a compelling clinical need for robust and reliable biomarkers of VTA-DMN functional connectivity. A major goal for future research will be to determine if such a biomarker can be validated on $3 \mathrm{~T}$ scans that are used for clinical purposes.

Beyond CL and VTA, our exploratory analyses suggest that the DMN has subcortical connections in additional regions of the brainstem, hypothalamus, thalamus, basal ganglia, and basal forebrain. These findings should be considered hypothesis-generating and will require validation in future connectivity studies. To inform the design of future experiments, we emphasize that several subcortical nuclei that demonstrated DMN correlations have strong data to support their role in modulating arousal, and hence consciousness, in prior animal studies. In particular, DR and MnR have been shown in animal electrophysiological experiments to regulate arousal (McGinty and Harper, 1976; Trulson and Jacobs, 1979; Xi et al., 2004), and these serotonergic nuclei in the pontomesencephalic raphe have been shown to be functionally connected to the DMN (Bär et al., 2016) or to constitute a sub-network of the DMN (Beliveau et al., 2015) in prior human rs-fMRI experiments. The mRt and PnO nuclei, classically considered the brainstem's "reticular core", are also recognized as key nodes of an ascending reticular activating system, based on decades of electrophysiologic investigations of arousal in animal models (Moruzzi and Magoun, 1949; Steriade et al., 1982; Xi et al., 2004). Similarly, our observation of lateral hypothalamic area and tuberomammillary nucleus connectivity with the DMN is consistent with electrophysiologic studies in rodent models of sleepwake cycle regulation (Alam et al., 2002; Takahashi et al., 2006). Collectively, these exploratory results thus add to a strong body of evidence in animal models, and a small but growing body of evidence in human studies, that multiple subcortical regions are involved in arousal regulation. Our findings expand upon prior studies by suggesting that DMN functional connectivity is a mechanism by which these subcortical nuclei activate the cerebral cortex to promote consciousness. Elucidation of the precise physiological mechanisms, temporal dynamics, and anatomic subspecialization of these subcortico-cortical connections will require multi-modality investigations of arousal in animal models (Pais-Roldán et al., 2019) and human experiments (Fultz et al., 2019) designed to interrogate the structure and function of subcortical arousal pathways at increasingly high levels of spatial and temporal resolution (DeFelipe, 2010).

When averaged over data from many subjects, the NASCAR and seed-based correlation methods yielded similar spatial patterns and contrast of the subcortical DMN. There has been debate about whether global signal regression helps or hurts the correlation analysis (Murphy and Fox, 2017). Without global signal regression, the correlation measures tend to be inflated due to the involvement of global "physiological" signals (Chen et al., 2020). On the other hand, the application of global signal regression introduces negative correlations (Murphy et al., 2009; Murphy and Fox, 2017). In contrast, NASCAR directly models the "global physiological" network as one of the low-rank components. In fact, this component was identified as 
the first network during the tensor decomposition, with the network strength $\lambda$ even higher than that of the DMN. In this way, NASCAR successfully decoupled the DMN from this global component, avoiding the ambiguity in the interpretation of the seed-based correlation results.

A manifestation of this ambiguity generated by seed-based correlation with global signal regression is seen in the VTA functional connectivity results. Whereas the NASCAR method detected DMN correlations within the VTA, the seed-based correlation with global signal regression detected correlations and anti-correlations. Prior studies have shown that the VTA contains not only dopaminergic neurons, but also GABAergic and glutamatergic neurons, raising the possibility that VTA interactions with the DMN could be spatially heterogeneous. However, prior neuronal labeling studies in rodents, non-human primates, and humans (Breton et al., 2019; Root et al., 2016; Taylor et al., 2014) indicate that dopaminergic, GABAergic, and glutamatergic neurons are intermingled within the VTA, making it unlikely that there would be discrete subregions of BOLD correlations and anticorrelations within the VTA. Furthermore, our immunostaining results (Fig. 9) revealed a symmetric, spatially contiguous distribution of dopaminergic neurons within the VTA. Given that the majority of VTA neurons produce dopamine (Taylor et al., 2014), these immunostaining results suggest that the spatially contiguous correlation results generated by the NASCAR method are more anatomically plausible than the spatially disparate correlation and anti-correlation results generated by the seed-based correlation with global signal regression.

A limitation of the NASCAR approach is the assumption of perfect inter-subject coregistration. The low-rank tensor model would fail if there were substantial spatial misalignment among subjects. In this study, we relied on the boundary-based registration method (Greve and Fischl, 2009) used in the HCP minimal preprocessing pipeline (Glasser et al., 2013). Although many model-based and more recently deep-learning-based approaches (Balakrishnan et al., 2019; Cheng et al., 2020; Robinson et al., 2014; Yeo et al., 2010) have been proposed to improve inter-subject coregistration, registration of subcortical regions remains challenging, especially when working with fMRI data where the spatial resolution is insufficient. For these reasons, although our results shown in Figs. 2 and 3 exhibited neuroanatomically plausible patterns of the subcortical DMN, we caution against making inferences on the voxel level, particularly near the boundaries of subcortical structures. The functional connectivity map in supplementary Fig. S1 shows the functional connectivity results before interpolation and illustrates this limitation.

As with other data-driven approaches (e.g., ICA), post-hoc manual inspection of the decomposed components is required. Theoretically, one NASCAR component may contain multiple networks, or multiple NASCAR components may represent sub-networks of a large-scale canonical network. However, in this work, the DMN was identified as the second component and confirmed by visualization of the cortical map spatially shown in Fig. 1 (e). We did not find other components exhibiting a spatial map similar to the DMN. Due to this data-driven property of the NASCAR decomposition method, exploration of other components besides the DMN is a promising future direction.

The SNR of fMRI data is very low, as all 30 networks extracted using the NASCAR method accumulatively could only explain $~ 11 \%$ of the variance in the data. Moreover, the SNR in subcortical regions is much lower than that in the cerebral cortex, making it difficult to identify functional connectivity in subcortical regions, particularly in the brainstem (Sclocco et al., 2018). As seen in Fig. 4, the average magnitude of DMN signals identified in the subcortical regions is only $\sim 5 \%$ of that in the cortical nodes. We attempted to address this inherent limitation of subcortical fMRI data by analyzing the 7T HCP rs-fMRI dataset, which has possibly the best SNR of any publicly available dataset. Hence, it is difficult to judge whether the results discovered in this work will be reproducible when the method is applied to datasets with lower SNR, e.g., HCP 3T or clinical 3T scans. Reproducing and generalizing these findings to $3 \mathrm{~T}$ scanners and lower resolution rs-fMRI datasets is essential for clinical translation and is a key goal for future investigation.

\section{Conclusion}

We provide a functional connectivity map of the subcortical DMN in the human brain. We reveal new functional connectivity properties of the brainstem, hypothalamus, thalamus, and basal forebrain, which may be used in future investigations of subcortical contributions to human consciousness. The subcortical DMN connectivity map may also be used in clinical trials as a predictive biomarker to inform patient selection or as a pharmacodynamic biomarker to measure whether a therapy engages its target within the DMN. We release the subcortical DMN connectivity map via Lead-DBS, FreeSurfer and OpenNEURO platforms for use in future neuromodulation studies.

\section{Data and code availability}

The data used in this study are publicly available from the Wash U/U Minn component of the Human Connectome Project, Young Adult Study at https://www.humanconnectome.org/study/hcp-young-adult.

For research purposes, we release the subcortical functional map of the default mode network at Lead-DBS (https://www.lead-dbs.org/help support/knowledge-base/atlasesresources/atlases), FreeSurfer (https://surfer.nmr.mgh.harvard.edu), and OpenNeURo (https://openneuro.org/data

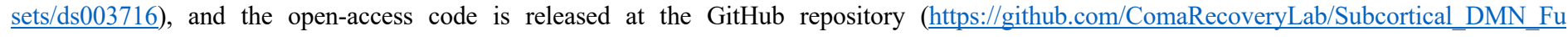
nctional Connectivity).

\section{Disclosure of competing Interest}

BF has a financial interest in CorticoMetrics, a company whose medical pursuits focus on brain imaging and measurement technologies. BF's interests were reviewed and are managed by Massachusetts General Hospital and Partners HealthCare in accordance with their conflict of interest policies. 
bioRxiv preprint doi: https://doi.org/10.1101/2021.07.13.452265; this version posted July 14, 2021. The copyright holder for this preprint (which was not certified by peer review) is the author/funder, who has granted bioRxiv a license to display the preprint in perpetuity. It is made available under aCC-BY 4.0 International license.

\section{Acknowledgment}

This study was supported by the NIH Director's Office (DP2HD101400), National Institute of Neurological Disorders and Stroke (R21NS109627, RF1NS115268, R01NS0525851, R21NS072652, R01NS070963, R01NS083534, U01NS086625, U24NS10059103, R01NS105820), BRAIN Initiative Cell Census Network (U01MH1 17023), National Institute for Biomedical Imaging and Bioengineering (P41EB015896, R01EB023281, R01EB006758, R21EB018907, R01EB019956, P41EB030006), National Institute on Aging (R56AG064027, R01AG064027, R01AG008122, R01AG016495), National Institute of Mental Health (R01MH123195, R01MH121885, 1RF1MH123195), the James S. McDonnell Foundation, and the Tiny Blue Dot Foundation. This work was also made possible by the resources provided by Shared Instrumentation Grants S10RR023401, S10RR019307, and S10RR023043. Additional support was provided by the NIH Blueprint for Neuroscience Research (U01MH093765), part of the multi-institutional Human Connectome Project.

\section{References}

Akrami, H., Joshi, A.A., Li, J., Leahy, R.M., 2019. Group-wise alignment of resting fMRI in space and time, in: Angelini, E.D., Landman, B.A. (Eds.), Medical Imaging 2019: Image Processing. Presented at the Image Processing, SPIE, San Diego, United States, p. 103. https://doi.org/10.1117/12.2512564

Alam, Md.N., Gong, H., Alam, T., Jaganath, R., McGinty, D., Szymusiak, R., 2002. Sleep-waking discharge patterns of neurons recorded in the rat perifornical lateral hypothalamic area. The Journal of Physiology 538, 619-631. https://doi.org/10.1113/jphysiol.2001.012888

Alves, P.N., Foulon, C., Karolis, V., Bzdok, D., Margulies, D.S., Volle, E., Thiebaut de Schotten, M., 2019. An improved neuroanatomical model of the default-mode network reconciles previous neuroimaging and neuropathological findings. Commun Biol 2, 370. https://doi.org/10.1038/s42003-019-0611-3

Baker, J.L., Ryou, J.-W., Wei, X.F., Butson, C.R., Schiff, N.D., Purpura, K.P., 2016. Robust modulation of arousal regulation, performance, and frontostriatal activity through central thalamic deep brain stimulation in healthy nonhuman primates. Journal of Neurophysiology 116, 2383-2404. https://oi.org/10.1152/jn.01129.2015

Balakrishnan, G., Zhao, A., Sabuncu, M.R., Guttag, J., Dalca, A.V., 2019. VoxelMorph: A learning framework for deformable medical image registration. IEEE Trans Med. Imaging 38, 1788-1800. https://doi.org/10.1109/TMI.2019.2897538

Bär, K.-J., de la Cruz, F., Schumann, A., Koehler, S., Sauer, H., Critchley, H., Wagner, G., 2016. Functional connectivity and network analysis of midbrain and brainstem nuclei. NeuroImage 134, 53-63. https://doi.org/10.1016/j.neuroimage.2016.03.071

Bar-Gad, I., Morris, G., Bergman, H., 2003. Information processing, dimensionality reduction and reinforcement learning in the basal ganglia. Progress in Neurobiology 71, 439-473. https://doi.org/10.1016/j.pneurobio.2003.12.001

Beliveau, V., Svarer, C., Frokjaer, V.G., Knudsen, G.M., Greve, D.N., Fisher, P.M., 2015. Functional connectivity of the dorsal and median raphe nuclei at rest. NeuroImage 116, 187-195. https://doi.org/10.1016/j.neuroimage.2015.04.065

Bhushan, C., Chong, M., Choi, S., Joshi, A.A., Haldar, J.P., Damasio, H., Leahy, R.M., 2016. Temporal non-local means filtering reveals real-time whole-brain cortical interactions in resting fMRI. PLoS ONE 11, e0158504. https://doi.org/10.1371/journal.pone.0158504

Blouw, P., Solodkin, E., Thagard, P., Eliasmith, C., 2016. Concepts as semantic pointers: A framework and computational model. Cogn Sci 40, $1128-1162$. https://doi.org/10.1111/cogs. 12265

Bodien, Y., Chatelle, C., Edlow, B., 2017. Functional Networks in Disorders of Consciousness. Semin Neurol 37, 485-502. https://doi.org/10.1055/s-0037-1607310

Bodien, Y.G., Threlkeld, Z.D., Edlow, B.L., 2019. Default mode network dynamics in covert consciousness. Cortex 119, 571-574. https://doi.org/10.1016/j.cortex.2019.01.014

Bota, M., Sporns, O., Swanson, L.W., 2015. Architecture of the cerebral cortical association connectome underlying cognition. Proc Natl Acad Sci USA 112, E2093E2101. https://doi.org/10.1073/pnas. 1504394112

Breton, J.M., Charbit, A.R., Snyder, B.J., Fong, P.T.K., Dias, E.V., Himmels, P., Lock, H., Margolis, E.B., 2019. Relative contributions and mapping of ventral tegmental area dopamine and GABA neurons by projection target in the rat. J Comp Neurol 527, 916-941. https://doi.org/10.1002/cne.24572

Brett, M., Penny, W., Kiebel, S., 2003. Introduction to random field theory. Human brain function 2.

Buckner, R.L., DiNicola, L.M., 2019. The brain's default network: updated anatomy, physiology and evolving insights. Nat Rev Neurosci 20 , 593-608. https://doi.org/10.1038/s41583-019-0212-7

Cain, J.A., Spivak, N.M., Coetzee, J.P., Crone, J.S., Johnson, M.A., Lutkenhoff, E.S., Real, C., Buitrago-Blanco, M., Vespa, P.M., Schnakers, C., Monti, M.M., 2021a. Ultrasonic thalamic stimulation in chronic disorders of consciousness. Brain Stimulation 14, 301-303. https://doi.org/10.1016/j.brs.2021.01.008

Cain, J.A., Visagan, S., Johnson, M.A., Crone, J., Blades, R., Spivak, N.M., Shattuck, D.W., Monti, M.M., 2021b. Real time and delayed effects of subcortical low intensity focused ultrasound. Sci Rep 11, 6100. https://doi.org/10.1038/s41598-021-85504-y

Calhoun, V.D., Adali, T., Pearlson, G.D., Pekar, J.J., 2001. A method for making group inferences from functional MRI data using independent component analysis. Hum. Brain Mapp. 14, 140-151. https://doi.org/10.1002/hbm.1048

Chen, J.E., Lewis, L.D., Chang, C., Tian, Q., Fultz, N.E., Ohringer, N.A., Rosen, B.R., Polimeni, J.R., 2020. Resting-state "physiological networks.” NeuroImage 213, 116707. https://doi.org/10.1016/j.neuroimage.2020.116707

Cheng, J., Dalca, A.V., Fischl, B., Zöllei, L., 2020. Cortical surface registration using unsupervised learning. NeuroImage 221, 117161. https://doi.org/10.1016/j.neuroimage.2020.117161

Choi, E.Y., Yeo, B.T.T., Buckner, R.L., 2012. The organization of the human striatum estimated by intrinsic functional connectivity. Journal of Neurophysiology 108, 2242-2263. https://doi.org/10.1152/jn.00270.2012

Corazzol, M., Lio, G., Lefevre, A., Deiana, G., Tell, L., André-Obadia, N., Bourdillon, P., Guenot, M., Desmurget, M., Luauté, J., Sirigu, A., 2017. Restoring consciousness with vagus nerve stimulation. Current Biology 27, R994-R996. https://doi.org/10.1016/j.cub.2017.07.060

Cunningham, S.I., Tomasi, D., Volkow, N.D., 2017. Structural and functional connectivity of the precuneus and thalamus to the default mode network: PrecuneusThalamus Connectivity to the DMN. Hum. Brain Mapp. 38, 938-956. https://doi.org/10.1002/hbm.23429

DeFelipe, J., 2010. From the connectome to the synaptome: An epic love story. Science 330, 1198-1201. https://doi.org/10.1126/science.1193378

Demertzi, A., Antonopoulos, G., Heine, L., Voss, H.U., Crone, J.S., de Los Angeles, C., Bahri, M.A., Di Perri, C., Vanhaudenhuyse, A., Charland-Verville, V., Kronbichler, M., Trinka, E., Phillips, C., Gomez, F., Tshibanda, L., Soddu, A., Schiff, N.D., Whitfield-Gabrieli, S., Laureys, S., 2015. Intrinsic functional connectivity differentiates minimally conscious from unresponsive patients. Brain 138, 2619-2631. https://doi.org/10.1093/brain/awv169

Dempsey, E.W., Morison, R.S., 1941. The production of rhythmically recurrent cortical potentials after localized thalamic stimulation. American Journal of PhysiologyLegacy Content 135, 293-300. https://doi.org/10.1152/ajplegacy.1941.135.2.293

Di Martino, A., Scheres, A., Margulies, D.S., Kelly, A.M.C., Uddin, L.Q., Shehzad, Z., Biswal, B., Walters, J.R., Castellanos, F.X., Milham, M.P., 2008. Functional connectivity of human striatum: A resting state fMRI study. Cerebral Cortex 18, 2735-2747. https://doi.org/10.1093/cercor/bhn041

Eban-Rothschild, A., Rothschild, G., Giardino, W.J., Jones, J.R., de Lecea, L., 2016. VTA dopaminergic neurons regulate ethologically relevant sleep-wake behaviors. Nat Neurosci 19, 1356-1366. https://doi.org/10.1038/nn.4377 
bioRxiv preprint doi: https://doi.org/10.1101/2021.07.13.452265; this version posted July 14, 2021. The copyright holder for this preprint (which was not certified by peer review) is the author/funder, who has granted bioRxiv a license to display the preprint in perpetuity. It is made available under aCC-BY 4.0 International license.

Edlow, B.L., Barra, M.E., Zhou, D.W., Foulkes, A.S., Snider, S.B., Threlkeld, Z.D., Chakravarty, S., Kirsch, J.E., Chan, S., Meisler, S.L., Bleck, T.P., Fins, J.J., Giacino, J.T., Hochberg, L.R., Solt, K., Brown, E.N., Bodien, Y.G., 2020. Personalized connectome mapping to guide targeted therapy and promote recovery of consciousness in the intensive care unit. Neurocrit Care 33, 364-375. https://doi.org/10.1007/s12028-020-01062-7

Edlow, B.L., Claassen, J., Schiff, N.D., Greer, D.M., 2021a. Recovery from disorders of consciousness: mechanisms, prognosis and emerging therapies. Nat Rev Neurol 17, 135-156. https://doi.org/10.1038/s41582-020-00428-x

Edlow, B.L., Mareyam, A., Horn, A., Polimeni, J.R., Witzel, T., Tisdall, M.D., Augustinack, J.C., Stockmann, J.P., Diamond, B.R., Stevens, A., Tirrell, L.S., Folkerth, R.D., Wald, L.L., Fischl, B., van der Kouwe, A., 2019. 7 Tesla MRI of the ex vivo human brain at 100 micron resolution. Sci Data 6, 244. https://doi.org/10.1038/s41597-019-0254-8

Edlow, B.L., Sanz, L.R.D., Polizzotto, L., Pouratian, N., Rolston, J.D., Snider, S.B., Thibaut, A., Stevens, R.D., Gosseries, O., the Curing Coma Campaign and its contributing members, Akbari, Y., Bleck, T.P., Diringer, M.N., Foreman, B., Hartings, J.A., Helbok, R., Hemphill, J.C., Ling, G.S.F., Mayer, S.A., McNett, M., Monti, M.M., Olson, D.M., Owen, A.M., Park, S., Provencio, J.J., Puybasset, L., Vespa, P., Wagner, A., Whyte, J., Ziai, W., 2021b. Therapies to restore consciousness in patients with severe brain injuries: A gap analysis and future directions. Neurocrit Care 35, 68-85. https://doi.org/10.1007/s12028-021-01227-y

Edlow, B.L., Takahashi, E., Wu, O., Benner, T., Dai, G., Bu, L., Grant, P.E., Greer, D.M., Greenberg, S.M., Kinney, H.C., Folkerth, R.D., 2012. Neuroanatomic connectivity of the human ascending arousal system critical to consciousness and its disorders. J Neuropathol Exp Neurol 71, 531-546. https://doi.org/10.1097/NEN.0b013e3182588293

Elias, G.J.B., Loh, A., Gwun, D., Pancholi, A., Boutet, A., Neudorfer, C., Germann, J., Namasivayam, A., Gramer, R., Paff, M., Lozano, A.M., 2020. Deep brain stimulation of the brainstem. Brain awaa374. https://doi.org/10.1093/brain/awaa374

Fischl, B., 2012. FreeSurfer. NeuroImage 62, 774-781. https://doi.org/10.1016/j.neuroimage.2012.01.021

Fox, M.D., Buckner, R.L., Liu, H., Chakravarty, M.M., Lozano, A.M., Pascual-Leone, A., 2014. Resting-state networks link invasive and noninvasive brain stimulation across diverse psychiatric and neurological diseases. Proceedings of the National Academy of Sciences 111, E4367-E4375. https://doi.org/10.1073/pnas.1405003111

Fox, M.D., Snyder, A.Z., Vincent, J.L., Corbetta, M., Van Essen, D.C., Raichle, M.E., 2005. The human brain is intrinsically organized into dynamic, anticorrelated functional networks. Proceedings of the National Academy of Sciences 102, 9673-9678. https://doi.org/10.1073/pnas.0504136102

Fox, M.D., Zhang, D., Snyder, A.Z., Raichle, M.E., 2009. The global signal and observed anticorrelated resting state brain networks. Journal of Neurophysiology 101, 3270-3283. https://doi.org/10.1152/jn.90777.2008

Fransson, P., 2005. Spontaneous low-frequency BOLD signal fluctuations: An fMRI investigation of the resting-state default mode of brain function hypothesis. Hum. Brain Mapp. 26, 15-29. https://doi.org/10.1002/hbm.20113

Fridman, E.A., Beattie, B.J., Broft, A., Laureys, S., Schiff, N.D., 2014. Regional cerebral metabolic patterns demonstrate the role of anterior forebrain mesocircuit dysfunction in the severely injured brain. Proceedings of the National Academy of Sciences 111, 6473-6478. https://doi.org/10.1073/pnas.1320969111

Fridman, E.A., Krimchansky, B.Z., Bonetto, M., Galperin, T., Gamzu, E.R., Leiguarda, R.C., Zafonte, R., 2010. Continuous subcutaneous apomorphine for severe disorders of consciousness after traumatic brain injury. Brain Injury 24, 636-641. https://doi.org/10.3109/02699051003610433

Fridman, E.A., Osborne, J.R., Mozley, P.D., Victor, J.D., Schiff, N.D., 2019. Presynaptic dopamine deficit in minimally conscious state patients following traumatic brain injury. Brain 142, 1887-1893. https://doi.org/10.1093/brain/awz118

Friston, K.J., Fletcher, P., Josephs, O., Holmes, A., Rugg, M., Turner, R., 1998. Event-related fMRI: characterizing differential responses. Neuroimage 7, $30-40$.

Fultz, N.E., Bonmassar, G., Setsompop, K., Stickgold, R.A., Rosen, B.R., Polimeni, J.R., Lewis, L.D., 2019. Coupled electrophysiological, hemodynamic, and cerebrospinal fluid oscillations in human sleep. Science 366, 628-631. https://doi.org/10.1126/science.aax5440

Giacino, J.T., Whyte, J., Bagiella, E., Kalmar, K., Childs, N., Khademi, A., Eifert, B., Long, D., Katz, D.I., Cho, S., Yablon, S.A., Luther, M., Hammond, F.M., Nordenbo, A., Novak, P., Mercer, W., Maurer-Karattup, P., Sherer, M., 2012. Placebo-controlled trial of amantadine for severe traumatic brain injury. N Engl J Med 366, 819826. https://doi.org/10.1056/NEJMoa1102609

Glasser, M.F., Sotiropoulos, S.N., Wilson, J.A., Coalson, T.S., Fischl, B., Andersson, J.L., Xu, J., Jbabdi, S., Webster, M., Polimeni, J.R., Van Essen, D.C., Jenkinson, M., 2013. The minimal preprocessing pipelines for the Human Connectome Project. NeuroImage 80, 105-124. https://doi.org/10.1016/j.neuroimage.2013.04.127

Gratwicke, J., Kahan, J., Zrinzo, L., Hariz, M., Limousin, P., Foltynie, T., Jahanshahi, M., 2013. The nucleus basalis of Meynert: A new target for deep brain stimulation in dementia? Neuroscience \& Biobehavioral Reviews 37, 2676-2688. https://doi.org/10.1016/j.neubiorev.2013.09.003

Greve, D.N., Fischl, B., 2009. Accurate and robust brain image alignment using boundary-based registration. NeuroImage 48, 63-72. https://doi.org/10.1016/j.neuroimage.2009.06.060

Guldenmund, P., Demertzi, A., Boveroux, P., Boly, M., Vanhaudenhuyse, A., Bruno, M.-A., Gosseries, O., Noirhomme, Q., Brichant, J.-F., Bonhomme, V., Laureys, S., Soddu, A., 2013. Thalamus, brainstem and salience network connectivity changes during propofol-induced sedation and unconsciousness. Brain Connectivity 3 , 273-285. https://doi.org/10.1089/brain.2012.0117

HCP Wiki, 2020. HCP data release updates: Known issues and planned fixes [WWW Document]. URL https://wiki.humanconnectome.org/display/PublicData/HCP+Data+Release+Updates\%3A+Known+Issues+and+Planned +fixes

He, Y., Wang, M., Chen, X., Pohmann, R., Polimeni, J.R., Scheffler, K., Rosen, B.R., Kleinfeld, D., Yu, X., 2018. Ultra-slow single-vessel BOLD and CBV-based fMRI spatiotemporal dynamics and their correlation with neuronal intracellular calcium signals. Neuron 97, 925-939.e5. https://doi.org/10.1016/j.neuron.2018.01.025

Horn, A., Fox, M.D., 2020. Opportunities of connectomic neuromodulation. NeuroImage 221, 117180. https://doi.org/10.1016/j.neuroimage.2020.117180

Horn, A., Reich, M., Vorwerk, J., Li, N., Wenzel, G., Fang, Q., Schmitz-Hübsch, T., Nick1, R., Kupsch, A., Volkmann, J., Kühn, A.A., Fox, M.D., 2017. Connectivity predicts deep brain stimulation outcome in Parkinson disease: DBS outcome in PD. Ann Neurol. 82, 67-78. https://doi.org/10.1002/ana.24974

Horn, A., Wenzel, G., Irmen, F., Huebl, J., Li, N., Neumann, W.-J., Krause, P., Bohner, G., Scheel, M., Kühn, A.A., 2019. Deep brain stimulation induced normalization of the human functional connectome in Parkinson's disease. Brain 142, 3129-3143. https://doi.org/10.1093/brain/awz239

Iglesias, J.E., Insausti, R., Lerma-Usabiaga, G., Bocchetta, M., Van Leemput, K., Greve, D.N., van der Kouwe, A., Fischl, B., Caballero-Gaudes, C., Paz-Alonso, P.M., 2018. A probabilistic atlas of the human thalamic nuclei combining ex vivo MRI and histology. NeuroImage 183, 314-326. https://doi.org/10.1016/j.neuroimage.2018.08.012

Joshi, A.A., Chong, M., Li, J., Choi, S., Leahy, R.M., 2018. Are you thinking what I'm thinking? Synchronization of resting fMRI time-series across subjects. NeuroImage 172, 740-752. https://doi.org/10.1016/j.neuroimage.2018.01.058

Kakusa, B., Saluja, S., Dadey, D.Y.A., Barbosa, D.A.N., Gattas, S., Miller, K.J., Cowan, R.P., Kouyoumdjian, Z., Pouratian, N., Halpern, C.H., 2020. Electrophysiology and Structural Connectivity of the Posterior Hypothalamic Region: Much to Learn From a Rare Indication of Deep Brain Stimulation. Front. Hum. Neurosci. 14, 164. https://doi.org/10.3389/fnhum.2020.00164

Kenny, J.D., Taylor, N.E., Brown, E.N., Solt, K., 2015. Dextroamphetamine (but not atomoxetine) induces reanimation from general anesthesia: Implications for the roles of dopamine and norepinephrine in active emergence. PLoS ONE 10, e0131914. https://doi.org/10.1371/journal.pone.0131914

Koch, C., Massimini, M., Boly, M., Tononi, G., 2016. Neural correlates of consciousness: progress and problems. Nat Rev Neurosci 17, 307-321. https://doi.org/10.1038/nrn.2016.22

Lee, T.-W., Xue, S.-W., 2018. Functional connectivity maps based on hippocampal and thalamic dynamics may account for the default-mode network. Eur J Neurosci 47, 388-398. https://doi.org/10.1111/ejn.13828 
Li, J., Choi, S., Joshi, A.A., Wisnowski, J.L., Leahy, R.M., 2020a. Temporal non-local means filtering for studies of intrinsic brain connectivity from individual resting fMRI. Medical Image Analysis 61, 101635. https://doi.org/10.1016/j.media.2020.101635

Li, J., Choi, S., Joshi, A.A., Wisnowski, J.L., Leahy, R.M., 2018. Global PDF-based temporal non-local means filtering reveals individual differences in brain connectivity, in: 2018 IEEE 15th International Symposium on Biomedical Imaging (ISBI 2018). Presented at the 2018 IEEE 15th International Symposium on Biomedical Imaging (ISBI 2018), IEEE, Washington, DC, pp. 15-19. https://doi.org/10.1109/ISBI.2018.8363513

Li, J., Haldar, J.P., Mosher, J.C., Nair, D.R., Gonzalez-Martinez, J.A., Leahy, R.M., 2019a. Scalable and robust tensor decomposition of spontaneous stereotactic EEG data. IEEE Trans. Biomed. Eng. 66, 1549-1558. https://doi.org/10.1109/TBME.2018.2875467

Li, J., Joshi, A.A., Leahy, R.M., 2020b. A network-based approach to study of ADHD using tensor decomposition of resting state fMRI data, in: 2020 IEEE 17 th International Symposium on Biomedical Imaging (ISBI). Presented at the 2020 IEEE 17th International Symposium on Biomedical Imaging (ISBI), IEEE, Iowa City, IA, USA, pp. 1-5. https://doi.org/10.1109/ISBI45749.2020.9098584

Li, J., Leahy, R.M., 2017. Parameter selection for optimized non-local means filtering of task fMRI, in: 2017 IEEE 14th International Symposium on Biomedical Imaging (ISBI 2017). Presented at the 2017 IEEE 14th International Symposium on Biomedical Imaging (ISBI 2017), IEEE, Melbourne, Australia, pp. 476-480. https://doi.org/10.1109/ISBI.2017.7950564

Li, J., Mosher, J.C., Nair, D.R., Gonzalez-Martinez, J., Leahy, R.M., 2017. Robust tensor decomposition of resting brain networks in stereotactic EEG, in: 2017 51st Asilomar Conference on Signals, Systems, and Computers. Presented at the 2017 51st Asilomar Conference on Signals, Systems, and Computers, IEEE, Pacific Grove, CA, USA, pp. 1544-1548. https://doi.org/10.1109/ACSSC.2017.8335616

Li, J., Wisnowski, J.L., Joshi, A.A., Leahy, R.M., 2021. Robust brain network identification from multi-subject asynchronous fMRI data. NeuroImage $227,117615$. https://doi.org/10.1016/j.neuroimage.2020.117615

Li, J., Wisnowski, J.L., Joshi, A.A., Leahy, R.M., 2019b. Brain network identification in asynchronous task fMRI data using robust and scalable tensor decomposition, in: Angelini, E.D., Landman, B.A. (Eds.), Medical Imaging 2019: Image Processing. Presented at the Image Processing, SPIE, San Diego, United States, p. 22. https://doi.org/10.1117/12.2512684

Lu, J., Jhou, T.C., Saper, C.B., 2006. Identification of wake-active dopaminergic neurons in the ventral periaqueductal gray matter. Journal of Neuroscience $26,193-202$. https://doi.org/10.1523/JNEUROSCI.2244-05.2006

McGinty, D.J., Harper, R.M., 1976. Dorsal raphe neurons: depression of firing during sleep in cats. Brain Research 101, 569-575. https://doi.org/10.1016/00068993(76) $90480-7$

Monti, M.M., Schnakers, C., Korb, A.S., Bystritsky, A., Vespa, P.M., 2016. Non-invasive ultrasonic thalamic stimulation in disorders of consciousness after severe brain injury: A first-in-man report. Brain Stimulation 9, 940-941. https://doi.org/10.1016/j.brs.2016.07.008

Morales, M., Margolis, E.B., 2017. Ventral tegmental area: cellular heterogeneity, connectivity and behaviour. Nat Rev Neurosci 18 , 73-85. https://doi.org/10.1038/nrn.2016.165

Moruzzi, G., Magoun, H.W., 1949. Brain stem reticular formation and activation of the EEG. Electroencephalography and Clinical Neurophysiology 1 , 455-473. https://doi.org/10.1016/0013-4694(49)90219-9

Murphy, K., Birn, R.M., Handwerker, D.A., Jones, T.B., Bandettini, P.A., 2009. The impact of global signal regression on resting state correlations: Are anti-correlated networks introduced? NeuroImage 44, 893-905. https://doi.org/10.1016/j.neuroimage.2008.09.036

Murphy, K., Fox, M.D., 2017. Towards a consensus regarding global signal regression for resting state functional connectivity MRI. NeuroImage 154, 169-173. https://doi.org/10.1016/j.neuroimage.2016.11.052

Neudorfer, C., Germann, J., Elias, G.J.B., Gramer, R., Boutet, A., Lozano, A.M., 2020. A high-resolution in vivo magnetic resonance imaging atlas of the human hypothalamic region. Scientific Data 7, 305. https://doi.org/10.1038/s41597-020-00644-6

Norton, L., Hutchison, R.M., Young, G.B., Lee, D.H., Sharpe, M.D., Mirsattari, S.M., 2012. Disruptions of functional connectivity in the default mode network of comatose patients. Neurology 78, 175-181. https://doi.org/10.1212/WNL.0b013e31823fcd61

Oishi, Y., Suzuki, Y., Takahashi, K., Yonezawa, T., Kanda, T., Takata, Y., Cherasse, Y., Lazarus, M., 2017. Activation of ventral tegmental area dopamine neurons produces wakefulness through dopamine D2-like receptors in mice. Brain Struct Funct 222, 2907-2915. https://doi.org/10.1007/s00429-017-1365-7

Pais-Roldán, P., Edlow, B.L., Jiang, Y., Stelzer, J., Zou, M., Yu, X., 2019. Multimodal assessment of recovery from coma in a rat model of diffuse brainstem tegmentum injury. NeuroImage 189, 615-630. https://doi.org/10.1016/j.neuroimage.2019.01.060

Palmiter, R.D., 2011. Dopamine signaling as a neural correlate of consciousness. Neuroscience 198, 213-220. https://doi.org/10.1016/j.neuroscience.2011.06.089

Parent, A., Hazrati, L.-N., 1995. Functional anatomy of the basal ganglia. I. The cortico-basal ganglia-thalamo-cortical loop. Brain Research Reviews $20,91-127$. https://doi.org/10.1016/0165-0173(94)00007-C

Parvizi, J., 2001. Consciousness and the brainstem. Cognition 79, 135-160. https://doi.org/10.1016/S0010-0277(00)00127-X

Qin, P., Northoff, G., 2011. How is our self related to midline regions and the default-mode network? NeuroImage 57, 1221-1233. https://doi.org/10.1016/j.neuroimage.2011.05.028

Raichle, M.E., 2015. The brain's default mode network. Annu. Rev. Neurosci. 38, 433-447. https://doi.org/10.1146/annurev-neuro-071013-014030

Raichle, M.E., Snyder, A.Z., 2007. A default mode of brain function: A brief history of an evolving idea. NeuroImage 37, 1083-1090. https://doi.org/10.1016/j.neuroimage.2007.02.041

Redinbaugh, M.J., Phillips, J.M., Kambi, N.A., Mohanta, S., Andryk, S., Dooley, G.L., Afrasiabi, M., Raz, A., Saalmann, Y.B., 2020. Thalamus modulates consciousness via layer-specific control of cortex. Neuron 106, 66-75.e12. https://doi.org/10.1016/j.neuron.2020.01.005

Robinson, E.C., Jbabdi, S., Glasser, M.F., Andersson, J., Burgess, G.C., Harms, M.P., Smith, S.M., Van Essen, D.C., Jenkinson, M., 2014. MSM: A new flexible framework for multimodal surface matching. NeuroImage 100, 414-426. https://doi.org/10.1016/j.neuroimage.2014.05.069

Root, D.H., Wang, H.-L., Liu, B., Barker, D.J., Mód, L., Szocsics, P., Silva, A.C., Maglóczky, Z., Morales, M., 2016. Glutamate neurons are intermixed with midbrain dopamine neurons in nonhuman primates and humans. Sci Rep 6, 30615. https://doi.org/10.1038/srep30615

Schiff, N.D., 2010. Recovery of consciousness after brain injury: a mesocircuit hypothesis. Trends in Neurosciences 33, 1-9. https://doi.org/10.1016/j.tins.2009.11.002

Schiff, N.D., 2008. Central thalamic contributions to arousal regulation and neurological disorders of consciousness. Annals of the New York Academy of Sciences 1129 , 105-118. https://doi.org/10.1196/annals.1417.029

Schiff, N.D., Giacino, J.T., Kalmar, K., Victor, J.D., Baker, K., Gerber, M., Fritz, B., Eisenberg, B., O’Connor, J., Kobylarz, E.J., Farris, S., Machado, A., McCagg, C., Plum, F., Fins, J.J., Rezai, A.R., 2007. Behavioural improvements with thalamic stimulation after severe traumatic brain injury. Nature 448, 600-603. https://doi.org/10.1038/nature06041

Sclocco, R., Beissner, F., Bianciardi, M., Polimeni, J.R., Napadow, V., 2018. Challenges and opportunities for brainstem neuroimaging with ultrahigh field MRI. NeuroImage 168, 412-426. https://doi.org/10.1016/j.neuroimage.2017.02.052

Smith, S.M., Beckmann, C.F., Andersson, J., Auerbach, E.J., Bijsterbosch, J., Douaud, G., Duff, E., Feinberg, D.A., Griffanti, L., Harms, M.P., Kelly, M., Laumann, T., Miller, K.L., Moeller, S., Petersen, S., Power, J., Salimi-Khorshidi, G., Snyder, A.Z., Vu, A.T., Woolrich, M.W., Xu, J., Yacoub, E., Uğurbil, K., Van Essen, D.C., Glasser, M.F., 2013. Resting-state fMRI in the Human Connectome Project. NeuroImage 80, 144-168. https://doi.org/10.1016/j.neuroimage.2013.05.039

Solt, K., Cotten, J.F., Cimenser, A., Wong, K.F.K., Chemali, J.J., Brown, E.N., 2011. Methylphenidate actively induces emergence from general anesthesia. Anesthesiology 115, 791-803. https://doi.org/10.1097/ALN.0b013e31822e92e5 
Solt, K., Van Dort, C.J., Chemali, J.J., Taylor, N.E., Kenny, J.D., Brown, E.N., 2014. Electrical stimulation of the ventral tegmental area induces reanimation from general anesthesia. Anesthesiology 121, 311-319. https://doi.org/10.1097/ALN.0000000000000117

Steriade, M., Glenn, L.L., 1982. Neocortical and caudate projections of intralaminar thalamic neurons and their synaptic excitation from midbrain reticular core. Journal of Neurophysiology 48, 352-371. https://doi.org/10.1152/jn.1982.48.2.352

Steriade, M., Oakson, G., Ropert, N., 1982. Firing rates and patterns of midbrain reticular neurons during steady and transitional states of the sleep-waking cycle. Exp Brain Res 46, 37-51. https://doi.org/10.1007/BF00238096

Swanson, L.W., 2000. Cerebral hemisphere regulation of motivated behavior. Brain Research 886, 113-164. https://doi.org/10.1016/S0006-8993(00)02905-X

Takahashi, K., Lin, J.-S., Sakai, K., 2006. Neuronal activity of histaminergic tuberomammillary neurons during wake-sleep states in the mouse. Journal of Neuroscience 26, 10292-10298. https://doi.org/10.1523/JNEUROSCI.2341-06.2006

Taylor, N.E., Van Dort, C.J., Kenny, J.D., Pei, J., Guidera, J.A., Vlasov, K.Y., Lee, J.T., Boyden, E.S., Brown, E.N., Solt, K., 2016. Optogenetic activation of dopamine neurons in the ventral tegmental area induces reanimation from general anesthesia. Proc Natl Acad Sci USA 113, 12826-12831. https://doi.org/10.1073/pnas.1614340113

Taylor, S.R., Badurek, S., Dileone, R.J., Nashmi, R., Minichiello, L., Picciotto, M.R., 2014. GABAergic and glutamatergic efferents of the mouse ventral tegmental area: Mouse VTA projections. J. Comp. Neurol. 522, 3308-3334. https://doi.org/10.1002/cne.23603

Thibaut, A., Bruno, M.-A., Ledoux, D., Demertzi, A., Laureys, S., 2014. tDCS in patients with disorders of consciousness: Sham-controlled randomized double-blind study. Neurology 82, 1112-1118. https://doi.org/10.1212/WNL.0000000000000260

Thomas Yeo, B.T., Krienen, F.M., Sepulcre, J., Sabuncu, M.R., Lashkari, D., Hollinshead, M., Roffman, J.L., Smoller, J.W., Zöllei, L., Polimeni, J.R., Fischl, B., Liu, H., Buckner, R.L., 2011. The organization of the human cerebral cortex estimated by intrinsic functional connectivity. Journal of Neurophysiology $106,1125-1165$. https://doi.org/10.1152/jn.00338.2011

Trulson, M.E., Jacobs, B.L., 1979. Raphe unit activity in freely moving cats: Correlation with level of behavioral arousal. Brain Research 163, 135-150. https://doi.org/10.1016/0006-8993(79)90157-4

Trutti, A.C., Fontanesi, L., Mulder, M.J., Bazin, P.-L., Hommel, B., Forstmann, B.U., 2021. A probabilistic atlas of the human ventral tegmental area (VTA) based on 7 Tesla MRI data. Brain Struct Funct 226, 1155-1167. https://doi.org/10.1007/s00429-021-02231-w

Uddin, L.Q., Clare Kelly, A.M., Biswal, B.B., Xavier Castellanos, F., Milham, M.P., 2009. Functional connectivity of default mode network components: Correlation, anticorrelation, and causality. Hum. Brain Mapp. 30, 625-637. https://doi.org/10.1002/hbm.20531

Van der Werf, Y.D., Witter, M.P., Groenewegen, H.J., 2002. The intralaminar and midline nuclei of the thalamus. Anatomical and functional evidence for participation in processes of arousal and awareness. Brain Research Reviews 39, 107-140. https://doi.org/10.1016/S0165-0173(02)00181-9

Van Essen, D.C., Ugurbil, K., Auerbach, E., Barch, D., Behrens, T.E.J., Bucholz, R., Chang, A., Chen, L., Corbetta, M., Curtiss, S.W., Della Penna, S., Feinberg, D., Glasser, M.F., Harel, N., Heath, A.C., Larson-Prior, L., Marcus, D., Michalareas, G., Moeller, S., Oostenveld, R., Petersen, S.E., Prior, F., Schlaggar, B.L., Smith, S.M., Snyder, A.Z., Xu, J., Yacoub, E., 2012. The Human Connectome Project: A data acquisition perspective. NeuroImage 62, 2222-2231. https://doi.org/10.1016/j.neuroimage.2012.02.018

Villablanca, J.R., Marcus, R.J., Olmstead, C.E., 1976. Effects of caudate nuclei or frontal cortex ablations in cats. II. Sleep-wakefulness, EEG, and motor activity. Experimental Neurology 53, 31-50. https://doi.org/10.1016/0014-4886(76)90279-X

Wang, X., Xu, M., Song, Y., Li, X., Zhen, Z., Yang, Z., Liu, J., 2014. The network property of the thalamus in the default mode network is correlated with trait mindfulness. Neuroscience 278, 291-301. https://doi.org/10.1016/j.neuroscience.2014.08.006

Whyte, J., Rajan, R., Rosenbaum, A., Katz, D., Kalmar, K., Seel, R., Greenwald, B., Zafonte, R., Demarest, D., Brunner, R., Kaelin, D., 2014. Zolpidem and Restoration of Consciousness. American Journal of Physical Medicine \& Rehabilitation 93, 101-113. https://doi.org/10.1097/PHM.0000000000000069

Williams, S.T., Conte, M.M., Goldfine, A.M., Noirhomme, Q., Gosseries, O., Thonnard, M., Beattie, B., Hersh, J., Katz, D.I., Victor, J.D., Laureys, S., Schiff, N.D., 2013. Common resting brain dynamics indicate a possible mechanism underlying zolpidem response in severe brain injury. eLife 2 , e01157. https://doi.org/10.7554/eLife.01157

Worsley, K.J., Evans, A.C., Marrett, S., Neelin, P., 1992. A Three-dimensional statistical analysis for CBF activation studies in human brain. J Cereb Blood Flow Metab 12, 900-918. https://doi.org/10.1038/jcbfm.1992.127

Xi, M.-C., Morales, F.R., Chase, M.H., 2004. Interactions between GABAergic and cholinergic processes in the nucleus pontis oralis: Neuronal mechanisms controlling active (rapid eye movement) sleep and wakefulness. Journal of Neuroscience 24, 10670-10678. https://doi.org/10.1523/JNEUROSCI.1987-04.2004

Yeo, B.T.T., Sabuncu, M.R., Vercauteren, T., Ayache, N., Fischl, B., Golland, P., 2010. Spherical demons: Fast diffeomorphic landmark-free surface registration. IEEE Trans. Med. Imaging 29, 650-668. https://doi.org/10.1109/TMI.2009.2030797

Yetnikoff, L., Lavezzi, H.N., Reichard, R.A., Zahm, D.S., 2014. An update on the connections of the ventral mesencephalic dopaminergic complex. Neuroscience 282, 23-48. https://doi.org/10.1016/j.neuroscience.2014.04.010

Zhu, D.C., Tarumi, T., Khan, M.A., Zhang, R., 2015. Vascular coupling in resting-state fMRI: Evidence from multiple modalities. J Cereb Blood Flow Metab 35, 19101920. https://doi.org/10.1038/jcbfm.2015.166 